Article

\title{
Sexual Differentiation Is Coordinately Regulated by Cryptococcus neoformans CRK1 and GAT1
}

\author{
Kuang-Hung Liu@ and Wei-Chiang Shen * \\ Department of Plant Pathology and Microbiology, National Taiwan University, Taipei 10617, Taiwan; \\ david0517@gmail.com \\ * Correspondence: wcshen@ntu.edu.tw; Tel.: +886-2-3366-4599
}

Received: 17 May 2020; Accepted: 16 June 2020; Published: 19 June 2020

\begin{abstract}
The heterothallic basidiomycetous fungus Cryptococcus neoformans has two mating types, MATa and MAT $\alpha$. Morphological progression of bisexual reproduction in C. neoformans is as follows: yeast to hyphal transition, filament extension, basidium formation, meiosis, and sporulation. C. neoformans Cdk-related kinase 1 (CRK1) is a negative regulator of bisexual mating. In this study, we characterized the morphological features of mating structures in the crk1 mutant and determined the genetic interaction of $C R K 1$ in the regulatory networks of sexual differentiation. In the bilateral crk1 mutant cross, despite shorter length of filaments than in the wild-type cross, dikaryotic filaments and other structures still remained intact during bisexual mating, but the timing of basidium formation was approximately $18 \mathrm{~h}$ earlier than in the cross between wild type strains. Furthermore, gene expression analyses revealed that CRK1 modulated the expression of genes involved in the progression of hyphal elongation, basidium formation, karyogamy and meiosis. Phenotypic results showed that, although deletion of $C$. neoformans CRK1 gene increased the efficiency of bisexual mating, filamentation in the $c r k 1$ mutant was blocked by MAT2 or ZNF2 mutation. A bioinformatics survey predicted the C. neoformans GATA transcriptional factor Gat1 as a potential substrate of Crk1 kinase. Our genetic and phenotypic findings revealed that $C$. neoformans $G A T 1$ and $C R K 1$ formed a regulatory circuit to negatively regulate MAT2 to control filamentation progression and transition during bisexual mating.
\end{abstract}

Keywords: Cryptococcus neoformans; CRK1; GAT1; bisexual mating; filament differentiation

\section{Introduction}

Cryptococcus spp. are a group of basidiomycetous fungi that can be isolated from pigeon droppings, soil and trees and cause meningitis in humans [1,2]. Infection with two major Cryptococcus species, C. neoformans and C. gattii, results in cryptococcosis [3]. C. gattii is distributed in tropical or subtropical areas and infects immunocompetent individuals [4-6], whereas C. neoformans is the major human fungal pathogen that infects AIDS patients worldwide.

C. neoformans grows vegetatively as yeast cells and is a bipolar heterothallic fungus that has mating type MATa and MAT $\alpha$ cells. The life cycle of $C$. neoformans has been described [7]. Several environmental conditions, such as nitrogen starvation, room temperature, and the presence of mating pheromone, trigger bisexual differentiation and same-sex mating in C. neoformans. Sexual development in C. neoformans starts with a morphological transition from a yeast form to a filamentous hypha.

Like many basidiomycetes, C. neoformans undergoes sexual differentiation with four major events: cell fusion, dikaryotic filament formation, meiosis, and sporulation. When bisexual mating is initiated in C. neoformans, MATa and MAT $\alpha$ mating type cells secrete pheromones via the transporter Ste6 [8]. Pheromones are perceived by the pheromone receptors Ste3a and Ste3 $\alpha$, which activates the heterotrimeric $\mathrm{G}$ proteins (Gpa2, Gpb1 and Gpg2) and p21-activated kinase (PAK) family proteins $($ Ste20a/ $\alpha)$ [4,9-12]. Signals are transduced to the Cryptococcus protein kinase 1 (Cpk1) 
mitogen-activated protein kinase (MAPK) signaling cascade, including the core components Ste11a/ $\alpha$, Ste7, and Cpk1 [13-15]. C. neoformans MAT2 is a high-mobility-group (HMG) transcription factor acting downstream of the Cpk1 MAPK pathway and binds to the pheromone response element in the promoter region of mating specific genes to promote cell fusion $[5,16]$. The $C$. neoformans cell identity complex Sxi1 $\alpha /$ Sxi2a is activated by Mat2 directly and then induces CLP1 expression, which is needed for dikaryotic filament generation after cell fusion $[5,17,18]$. Znf2, a C2H2 zinc finger transcription factor, is also specifically required for dikaryotic filament formation but dispensable for cell fusion [16,19]. Znf2 regulates the expression of PUM1 and BRF1 for aerial hyphae formation and hyphal differentiation [19-22]. Additionally, C. neoformans Kar7 and Kar5 regulate karyogamy; Csa1, Csa2 and Ubc5 regulates meiosis, basidial maturation and sporulation [15,23-25].

Light can repress bisexual mating in C. neoformans via the Bwc1/Bwc2 complex by inhibiting the cell fusion stage and reducing dikaryotic filament generation [26,27]. Agrobacterium-mediated insertional mutagenesis was used to identify putative non-filamentation suppressors mediated by the Bwc1/Bwc2 complex [28]. C. neoformans CRK1 gene, a Saccharomyces cerevisiae IME2 homologue, was identified and characterized [29]. In S. cerevisiae, Ime2 is a meiotic-specific serine/threonine protein kinase that regulates meiosis and sporulation [30]. At the onset of meiosis, IME2 is induced by Ime1 to coordinate the follow-up meiotic progress [31,32]. The targets of $S$. cerevisiae Ime2, such as Sic1, Rfa2, Sum1, and Cdh1, are phosphorylated by Ime2 during meiosis. Other potential downstream targets of Ime2 were predicted with the Ime2 phosphoacceptor consensus sequence R-P-X-S/T-R/P/A [33-41].

The homologous protein kinases of Ime2 modulate different cellular functions in different fungal model systems. Neurospora crassa ime-2 gene plays a negative role in sexual differentiation. Mutation of N. crassa ime-2 increases the production of fruiting bodies in the presence of nitrogen. N. crassa vib-1, a transcription factor, regulates protoperithecia formation in N. crassa, which is epistatic to ime-2 in protoperithecia formation [42]. According to a study of the S. cerevisiae Ime2 consensus phosphorylation site, N. crassa VIB-1 contains the specific site RPRS ${ }^{* 60}$. Evidence also indicates that VIB-1 is an IME-2 phosphorylation target in N. crassa [42,43]. Ustilago maydis crk1, an S. cerevisiae IME2 homologous gene, is an MAPK member in the pheromone response pathway and controls morphogenesis. Recent study has found that $U$. maydis $C r k 1$ is required for sexual development and also related to early endosome motility during fungal infection [44-48].

Our prior study revealed that $C$. neoformans $C R K 1$ gene is a negative regulator of bisexual mating. The crk1 mutants show higher cell fusion efficiency and increased mating filamentation, and the timing for the formation of basidia and basidiospores was earlier than that seen in the wild-type cross. In this study, we detailed the morphological features of dikaryotic filaments in the bilateral crk1 mutant cross and conducted genetic and gene expression studies between C. neoformans CRK1 and MAT2 or ZNF2 in the bisexual mating process. We also identified that Gat1, a GATA type transcription factor, is a putative target of Crk1 and suggest that CRK1 and GAT1 may coordinately regulate bisexual mating in C. neoformans.

\section{Materials and Methods}

\subsection{Strains, Media and Growth Conditions}

C. neoformans serotype D strains listed in Table 1 were used. All strains were cultivated by standard media and handled according to previously published protocols and techniques [49]. Yeast-peptone-dextrose (YPD) medium was routinely used for culture maintenance at $30^{\circ} \mathrm{C}$, and V8 media incubated at $26^{\circ} \mathrm{C}$ were used for bisexual mating experiments, respectively. YPD medium with $200 \mu \mathrm{g} / \mathrm{mL}$ hygromycin B or with $100 \mu \mathrm{g} / \mathrm{mL}$ nourseothricin and $200 \mu \mathrm{g} / \mathrm{mL}$ hygromycin B was used for selecting transformants. 
Table 1. Cryptococcus neoformans strains used in this study.

\begin{tabular}{|c|c|c|}
\hline Strains & Description & Reference \\
\hline JEC20 & MATa & {$[50]$} \\
\hline JEC21 & $M A T \alpha$ & [50] \\
\hline JEC43 & $M A T \propto$ ura5 & [51] \\
\hline DLC2 & $M A T \alpha \operatorname{crk1::NAT^{\mathrm {R}}}$ & [29] \\
\hline DLC4 & MATa crk1::NAT ${ }^{\mathrm{R}}$ & [29] \\
\hline DLC18 & MATa crk1 + $\mathrm{P}_{G P D 1}:: G F P-H 2 B$ ura5 & [29] \\
\hline DLC21 & MATa $\mathrm{P}_{G P D 1}:: G F P-H 2 B$ ura5 & [29] \\
\hline DLC22 & $M A T \alpha c r k 1+\mathrm{P}_{G P D 1}:: C R K 1$ & This study \\
\hline DLC23 & MATa mat2::HYG ${ }^{\mathrm{R}}$ & This study \\
\hline DLC24 & $M A T \alpha$ mat2::HYG ${ }^{\mathrm{R}}$ & This study \\
\hline DLC25 & MATa mat $2:: H Y G^{\mathrm{R}}$ crk1::NAT ${ }^{\mathrm{R}}$ & This study \\
\hline DLC26 & MAT $\alpha$ mat2::HYG $G^{\mathrm{R}}$ crk1::NAT ${ }^{\mathrm{R}}$ & This study \\
\hline DLC27 & $M A T \alpha \mathrm{P}_{G P D 1}:: M A T 2$ & This study \\
\hline DLC28 & $M A T \mathbf{a} z n f 2:: H Y G^{\mathrm{R}}$ & This study \\
\hline DLC29 & $M A T \alpha z n f 2:: H Y G^{\mathrm{R}}$ & This study \\
\hline DLC30 & MATa znf2::HYG $G^{\mathrm{R}} c r k 1:: N A T^{\mathrm{R}}$ & This study \\
\hline DLC31 & $M A T \alpha z n f 2:: H Y G^{\mathrm{R}}$ crk1::NAT ${ }^{\mathrm{R}}$ & This study \\
\hline DLC32 & MATa gat $1:: H Y G^{\mathrm{R}}$ & This study \\
\hline DLC33 & $M A T \propto$ gat1::HYG ${ }^{\mathrm{R}}$ & This study \\
\hline DLC34 & MATa gat1 + GAT1::NAT ${ }^{\mathrm{R}}$ & This study \\
\hline DLC35 & $M A T \alpha$ gat1 + GAT1::NAT ${ }^{\mathrm{R}}$ & This study \\
\hline DLC36 & $M A T \alpha \mathrm{P}_{G P D 1}:: G A T 1$ & This study \\
\hline DLC37 & $M A T \alpha c r k 1+P_{G P D 1}: C R K 1$ & This study \\
\hline DLC38 & $M A T \alpha \operatorname{crk1}+\mathrm{P}_{\mathrm{GPD} 1}:: C R K 1+\mathrm{P}_{G P D 1}:: G A T 1$ & This study \\
\hline DLC39 & $M A T \mathbf{a} G A T 1^{\mathrm{T} 1164 \mathrm{~A}}:: H Y G^{\mathrm{R}}$ & This study \\
\hline DLC40 & $M A T \propto G A T 1^{\mathrm{T} 1164 \mathrm{~A}}:: H Y G^{\mathrm{R}}$ & This study \\
\hline DLC41 & MATa crk1::NAT ${ }^{\mathrm{R}}$ gat $1:: H Y G^{\mathrm{R}}$ & This study \\
\hline DLC42 & MAT $\alpha$ crk1::NAT ${ }^{\mathrm{R}}{ }_{\text {gat } 1:: H Y G^{\mathrm{R}}}$ & This study \\
\hline DLC43 & $M A T \mathbf{a} \mathrm{P}_{G P D 1}:: G A T 1$ & This study \\
\hline DLC44 & MATa GAT1 ${ }^{\mathrm{T} 1164 \mathrm{D}}:: H Y G^{\mathrm{R}}$ & This study \\
\hline DLC45 & $M A T \alpha G A T 1^{\mathrm{T} 1164 \mathrm{D}}:: H Y G^{\mathrm{R}}$ & This study \\
\hline DLC46 & MATa $c r k 1+G A T 1^{\mathrm{T} 1164 \mathrm{D}}:: H Y G^{\mathrm{R}}$ & This study \\
\hline DLC47 & $M A T \alpha c r k 1+G A T 1^{\mathrm{T} 1164 \mathrm{D}}:: H Y G^{\mathrm{R}}$ & This study \\
\hline DLC48 & MATa $\mathrm{P}_{G P D 1}:: C R K 1$ gat1::HYG ${ }^{\mathrm{R}}$ & This study \\
\hline DLC49 & $M A T \alpha \mathrm{P}_{G P D 1}:: C R K 1$ gat $1:: H Y G^{\mathrm{R}}$ & This study \\
\hline
\end{tabular}

\subsection{Construction of $C$. neoformans mat2 and znf2 Mutant Strains}

To delete MAT2 and ZNF2 genes in C. neoformans MATa and MAT $\alpha$ wild-type and crk1 mutant background, we generated the MAT2-HYG and ZNF2-HYG split markers for transformation by double-joint PCR as described [52,53]. The oligonucleotides used were listed in Table S1.

To generate MAT2-HYG split marker fragments, the 5'-and $3^{\prime}$-flanking regions of MAT2 were amplified with WC1130/WC1131 and WC1132/WC1133 primers, respectively. Then, 1.2-kb of the $5^{\prime}-H Y G$ cassette and $1.1 \mathrm{~kb}$ of the HYG-3' cassette, with a $~ 300-b p$ overlapped sequence, were amplified by PCR with primer pairs WC270/WC739 and WC765/WC271, respectively, with plasmid PJAF15 as DNA template. PCR products were purified, and $1 \mathrm{ng}$ of each product was used for double-joint PCR with primers WC1130/WC886 and WC1133/WC885, respectively, to generate $5^{\prime}$ and $3^{\prime}$ MAT2-HYG split marker fragments. The amplified fragments were separated by gel electrophoresis and purified for transformation.

To generate ZNF2-HYG split marker fragments, the $5^{\prime}$ - and $3^{\prime}$-flanking regions of ZNF2 were amplified with primer pairs WC1256/WC1257 and WC1258/WC1259, respectively. The $5^{\prime}$ and $3^{\prime}$ overlapped fragments of the HYG cassette were amplified as described previously. Double-joint PCR 
reactions were performed with primer pairs WC1256/WC886 and WC1259/WC885 to generate $5^{\prime}$ and 3' ZNF2-HYG split-marker fragments, respectively, for transformation.

For transformation, $1 \mu \mathrm{g}$ DNA of each $5^{\prime}$ and $3^{\prime}$ split-marker fragment was mixed and delivered into C. neoformans MATa and MAT $\alpha$ wild-type and crk1 mutant strains by particle bombardment. Transformants were selected on YPD medium containing $200 \mu / \mathrm{mL}$ hygromycin B and YPD medium containing $200 \mu \mathrm{g} / \mathrm{mL}$ hygromycin B and $100 \mu \mathrm{g} / \mathrm{mL}$ nourseothricin, and DNA was extracted by the FPFD method for PCR screening [54]. PCR reactions were performed using primer pairs WC875/WC876 and WC879/WC880 to screen for the presence of MAT2 and ZNF2. The mat2 deletion mutants failed to amplify the 120-bp wild-type MAT2 fragment and the znf2 mutants failed to amplify the 130-bp wild-type ZNF2 fragment.

\subsection{Overexpression of C. neoformans CRK1 in the MATa crk1 Mutant}

The CRK1 overexpression construct generated previously was used to deliver into MAT $\alpha$ crk1ura5 cells by biolistic transformation [29]. DNA was extracted from uracil prototrophic transformants by the FPFD method for PCR screening [54]. Candidate strains were further verified by real-time qRT-PCR assay.

\subsection{Overexpression of C. neoformans MAT2 in the MAT $\alpha$ Wild-type Strain}

To overexpress the C. neoformans MAT2 gene, PCR primers WC1734 and WC1735 were designed to amplify the $2.5-\mathrm{kb}$ genomic fragment containing the MAT2 open reading frame and downstream terminator sequence. PCR product was purified and cloned into pCR-Blunt II-TOPO vector (Invitrogen, Carlsbad, CA, USA). The positive clones were verified by sequencing and digested with BamHI and KpnI to release the MAT2 fragment. The fragment was then ligated with pYKL8, which contains a constitutive promoter of $C$. neoformans GPD1, to generate the MAT2 overexpression construct [27]. The construct was delivered into the MAT $\alpha$ ura5 strain JEC43 by biolistic transformation. Uracil prototrophic transformants were chosen and screened by PCR. Candidate clones were further verified by real-time qRT-PCR assay.

\subsection{Genetic Manipulation of C. neoformans GAT1 Gene}

To delete GAT1 in C. neoformans serotype D wild-type JEC20 and JEC21 strains, MATa crk1 and $M A T \alpha$ crk1 mutants, and MATa and MAT $\alpha \mathrm{P}_{G P D 1}:: C R K 1$ strains, we generated GAT1-HYG deletion split markers by using double-joint PCR. PCR was used to amplify $5^{\prime}$-and $3^{\prime}$-flanking sequences of GAT1 with primer pairs WC2432/WC2434 and WC2435/WC2437, respectively, with JEC21 genomic DNA. The $5^{\prime}$-and $3^{\prime}$-partial regions of the $H Y G$ cassette were amplified. The overlapped fragment containing GAT1 5'-flanking and 5'-HYG sequences was generated by double-joint PCR with primer pair WC2433/WC886. A similar amplification procedure with primer pair WC2436/WC885 was used to amplify the fragment containing GAT1 3'-flanking and HYG-3' sequences. Finally, $1 \mu \mathrm{g}$ of each split-marker fragment was mixed and delivered into $C$. neoformans serotype $\mathrm{D}$ strains by biolistic transformation. Transformants were selected on YPD containing $200 \mu \mathrm{g} / \mathrm{mL}$ hygromycin B and DNA was extracted by the FPFD method for PCR screening.

To reconstitute the wild-type GAT1 allele back into the mutant strains, the GAT1 locus was amplified from the JEC21 wild-type strain with primers WC2594 and WC2595. PCR fragment was purified and cloned into pCR-Blunt II-TOPO vector (Invitrogen, Carlsbad, CA, USA). The clones were digested by SacI and SpeI, and the inserted fragment was subcloned into $\mathrm{pCH} 233$ to generate the GAT1 reconstitution construct. The reconstitution plasmid was biolistically transformed into the gat1 deletion mutants. Stable transformants were selected on YPD medium containing $200 \mu \mathrm{g} / \mathrm{mL}$ hygromycin B and $100 \mu \mathrm{g} / \mathrm{mL}$ nourseothricin. The reconstituted strains were confirmed by PCR and southern blot analyses for the presence of GAT1 and subjected to phenotypic examination.

To overexpress GAT1, the open reading frame and downstream terminator region were amplified from JEC21 genomic DNA by PCR with primers WC2555 and WC2556 containing NotI site at the 
$5^{\prime}$ and $3^{\prime}$ ends, respectively. PCR product was purified and cloned into pCR-Blunt II-TOPO vector (Invitrogen, Carlsbad, CA, USA). The clones were verified by sequencing and digested with NotI to release the GAT1 fragment. Products were then ligated into pKHL1, which contains a constitutive promoter of $C$. neoformans GPD1 in pJAF15 vector [52], to generate the GAT1 overexpression plasmid. The construct was linearized by SacI, then introduced into the MATa and MAT $\alpha$ wild-type, and MAT $\alpha$ $c r k 1+P_{G P D 1}:: C R K 1$ strains by biolistic transformation. Transformants were selected on YPD medium containing $200 \mu \mathrm{g} / \mathrm{mL}$ hygromycin B, and DNA was extracted by the FPFD method for PCR screening [54]. Candidate clones were further verified by real-time qRT-PCR assay.

To generate the phospho-null and phospho-mimetic mutation in the predicted phosphorylation site of Gat1, site-directed mutagenesis was conducted by overlapping PCR with the mutated primer pairs WC2604/WC2605 and WC3257/WC3258, and GAT1-overexpression construct as a template. For phospho-null mutation, PCR primers WC2639/WC2605 and WC2604/WC1181 were used to amplify two fragments for overlapping; and for phospho-mimetic mutation, WC2639/WC3258 and WC3257/WC3258 primers were used. Overlapping PCR was conducted by using primers WC2567 and WC2573. A 2.3-kb overlapping fragment containing partial GAT1 open reading frame with GAT1 ${ }^{T 1164 A}$ and GAT1 ${ }^{T 1164 D}$ substitution and the terminator region was cut by BamHI and SpeI, and the fragment was purified and cloned into PCR-Blunt II-TOPO vector (Invitrogen, Carlsbad, CA, USA). Correct clones were confirmed by sequencing and the fragment with substituted amino acid was released by BamHI and SpeI and subcloned into pJAF15 to respectively generate the GAT1 ${ }^{T 1164 A}$ and GAT1 ${ }^{T 1164 D}$ site-directed mutagenesis construct with the HYG marker. The construct was linearized by BamHI and delivered into the MATa and MAT $\alpha$ wild-type cells or MATa crk1 and MAT $\alpha$ crk1 mutant strains by biolistic transformation. Stable transformants were selected on YPD medium containing $200 \mu \mathrm{g} / \mathrm{mL}$ hygromycin B, and DNA was extracted by the FPFD method and further subjected to PCR amplification and sequencing confirmation [54].

\subsection{Sample Preparation for Gene Expression Analyses}

Strains subjected to gene expression analyses in a- $\alpha$ opposite sex mating were grown in $5 \mathrm{~mL}$ YPD liquid medium at $30^{\circ} \mathrm{C}$ for overnight, then transferred to $45 \mathrm{~mL}$ liquid YPD medium and continuously grown for $22 \mathrm{~h}$ to reach the density at $1 \times 10^{8}$ cells/mL. Cells were harvested, washed with sterile water and resuspended in $5 \mathrm{~mL}$ sterile water. An equal amount of MATa and MAT $\alpha$ cells was mixed and $10 \mu \mathrm{L}$ of cell mixtures was spotted onto V8 agar medium and kept in the dark at $26^{\circ} \mathrm{C}$ to induce mating; part of the cell suspension was centrifuged, frozen and used as a $0 \mathrm{~h}$ control sample for gene expression analyses. Mating cells were harvested with sterile water from the surfaces of the plates at a designated time. All harvested cells were immediately frozen in liquid nitrogen and lyophilized for RNA extraction.

Total RNA was extracted by using TRIzol reagent as described (Invitrogen). $10 \mu \mathrm{g}$ of RNA from each sample was treated with Ambion ${ }^{\circledR}$ Turbo DNA-free ${ }^{\mathrm{TM}}$ kit (Invitrogen, Carlsbad, CA, USA) and single-strand cDNA was synthesized by using the High Capacity cDNA Reverse Transcription Kit (Applied Biosystems, Carlsbad, CA, USA ). Real-time PCR assay was performed with the Applied Biosystems StepOne real-time PCR system with KAPA SYBR FAST qPCR kits (Kapa Biosystems. Wilmington, MA, USA). C. neoformans GPD1 gene was used to normalize relative gene expression. All samples were conducted in triplicate for gene expression assay. Two-tailed Student's $t$ test ( $p$-value $<0.05)$ was used to compare mRNA levels between two samples. Primers used for real-time qRT-PCR experiments are listed in Supplementary Table S1.

\subsection{Fluorescence Microscopy}

Strains used for fluorescence microscopy were previously described [29]. For filament imaging, C. neoformans cells were spotted on SLAD agar medium containing $25 \mu \mathrm{g} / \mathrm{mL}$ calcofluor white and incubated at $26^{\circ} \mathrm{C}$ for $13 \mathrm{~h}$ under dark conditions, then collected by using $1 \mathrm{~mL}$ sterile water. An amount of $10 \mu \mathrm{L}$ cell suspension was spread on SLAD agar medium containing $25 \mu \mathrm{g} / \mathrm{mL}$ calcofluor white 
and covered with a coverslip. Photos were captured by using the DeltaVision system (GE Healthcare, Chicago, IL, USA) with an Olympus IX-71 inverted microscope equipped with a CoolSnap HQ2 high-resolution charged-coupled-device (CCD) camera and a 100× objective. The excitation and emission filter sets, CFP 438/24 and 470/24 and GFP/FITC 475/28 and 525/50, were used for observing calcofluor white and GFP, respectively.

\section{Results}

\subsection{In the C. neoformans Bilateral crk1 Mutant Cross, Dikaryotic Filamentation was Altered but Nucleus} Distribution Was Normal

C. neoformans CRK1 is a negative regulator of sexual reproduction. Sexual reproduction efficiency in the bilateral crk1 mutant cross is increased and the production of basidiospores occurs earlier than the wild-type cross. Furthermore, dikaryotic filaments at the edges of the mating colony are shorter in the bilateral crk1 mutant cross than the wild-type [29]. To further investigate the detailed developmental features in the bilateral crk1 mutant cross and whether nuclear distribution in the dikaryotic filaments of the mutant cross is affected, we used the GFP-H2B-tagged MATa wild-type and $c r k 1$ mutant strains, as previously described [29], to cross with the MAT $\alpha$ wild-type and crk1 mutant strains, respectively. Bisexual mating was monitored every $2 \mathrm{~h}$ up to $72 \mathrm{~h}$. On time-lapse observation, the length of dikaryotic filaments did not differ between the wild-type and bilateral crk1 mutant crosses at the edge of mating colonies at $16 \mathrm{~h}$ (Supplementary Figure S1). Two GFP-tagged nuclei were observed in each hyphal cell in both the wild-type and bilateral crk1 mutant crosses and the morphology of dikaryotic filaments was also no difference (Figure 1A,B). Furthermore, aerial hyphae differentiated to generate basidia in the bilateral crk1 mutant cross, and two GFP-labeled nuclei moved to the basidium at $18 \mathrm{~h}$ (Figure 1B). In contrast, in the wild-type, the morphology of dikaryotic filaments remained straight, with no basidia observed (Figure 1A). In the bilateral crk1 mutant cross, from 20 to $24 \mathrm{~h}$, the growth of dikaryotic filaments at the edge of mating colonies seemed to cease and the length was generally shorter when compared to the wild-type cross (Figure S1c-e). At the same time, GFP-labeled nuclei in the bilateral crk1 mutant cross underwent nuclear fusion and meiosis and generated four nuclei (Figure 1B); four long chains of basidiospores were apparently observed at $36 \mathrm{~h}$ and continuously until $72 \mathrm{~h}$ (Figure 1B). However, basidia emerged at $36 \mathrm{~h}$ in the wild type cross, then completed nuclear fusion and meiosis, and four long chains of basidiospores were observed at $48 \mathrm{~h}$ and up to $72 \mathrm{~h}$ (Figure 1A). Thus, during bisexual mating, the morphology of dikaryotic filaments and nuclei distribution did not differ between the wild-type and crk1 mutant. However, basidial formation, meiosis and the production of basidiospores were earlier in the bilateral crk1 mutant cross than the wild-type by approximately $18 \mathrm{~h}$.

Despite no change in the length of dikaryotic filaments between the wild-type and the bilateral crk1 mutant cross before $18 \mathrm{~h}$, afterward, two genotypes showed distinct lengths of dikaryotic filaments. Therefore, by using clamp cells stained with calcofluor white, we compared dikaryotic filaments between the wild-type and bilateral crk1 mutant crosses at 13 and $17 \mathrm{~h}$. The GFP-H2B-tagged MATa wild-type and $c r k 1$ strains were crossed with MAT $\alpha$ wild-type and $c r k 1$ mutant strains, respectively, on SLAD agar medium containing $25 \mu \mathrm{g} / \mathrm{mL}$ calcofluor white. The intact fused clamp cells emerged from dikaryotic filaments in both the wild-type and bilateral crk1 mutant cross at 13 and $17 \mathrm{~h}$ (Figure 2). At $13 \mathrm{~h}$, only one fused clamp was produced, and we measured the length from the tip of dikaryotic filaments to the junction of the yeast cell. After counting 120 dikaryotic filaments, which in the wild-type was a mean of $20.04 \pm 4.6 \mu \mathrm{m}$ and in the bilateral crk1 mutant cross was $19.57 \pm 3.73 \mu \mathrm{m}$. Then, at $17 \mathrm{~h}$, the extended and straight dikaryotic filaments generated two fused clamp cells in both the wild-type and bilateral crk1 mutant crosses. However, the length of dikaryotic filaments differed: the mean length in the wild-type was $80.1 \pm 7.22 \mu \mathrm{m}$ and in the bilateral crk1 mutant cross was $38 \pm 6.47 \mu \mathrm{m}$. Collectively, our detailed cytological characterization revealed that short dikaryotic filament phenotype exhibited by the crk1 mutant was due to reduced length of the hyphal segment after $13 \mathrm{~h}$ incubation but nuclear distribution and behavior were unaltered inside the mating structures, 
suggesting that progression of dikaryotic filamentation and further transition to basidium might be mediated by C. neoformans Crk1.

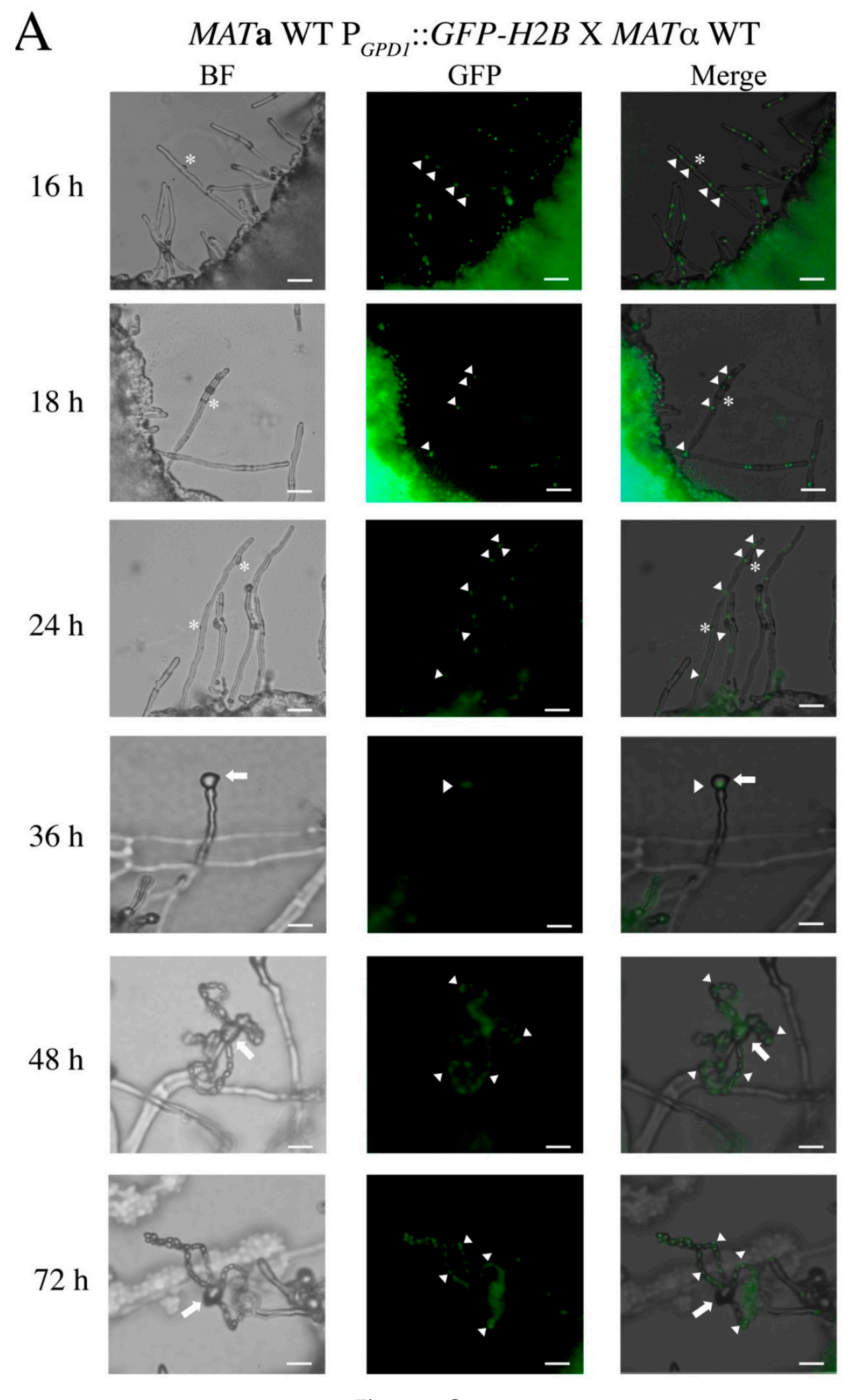

Figure 1. Cont. 

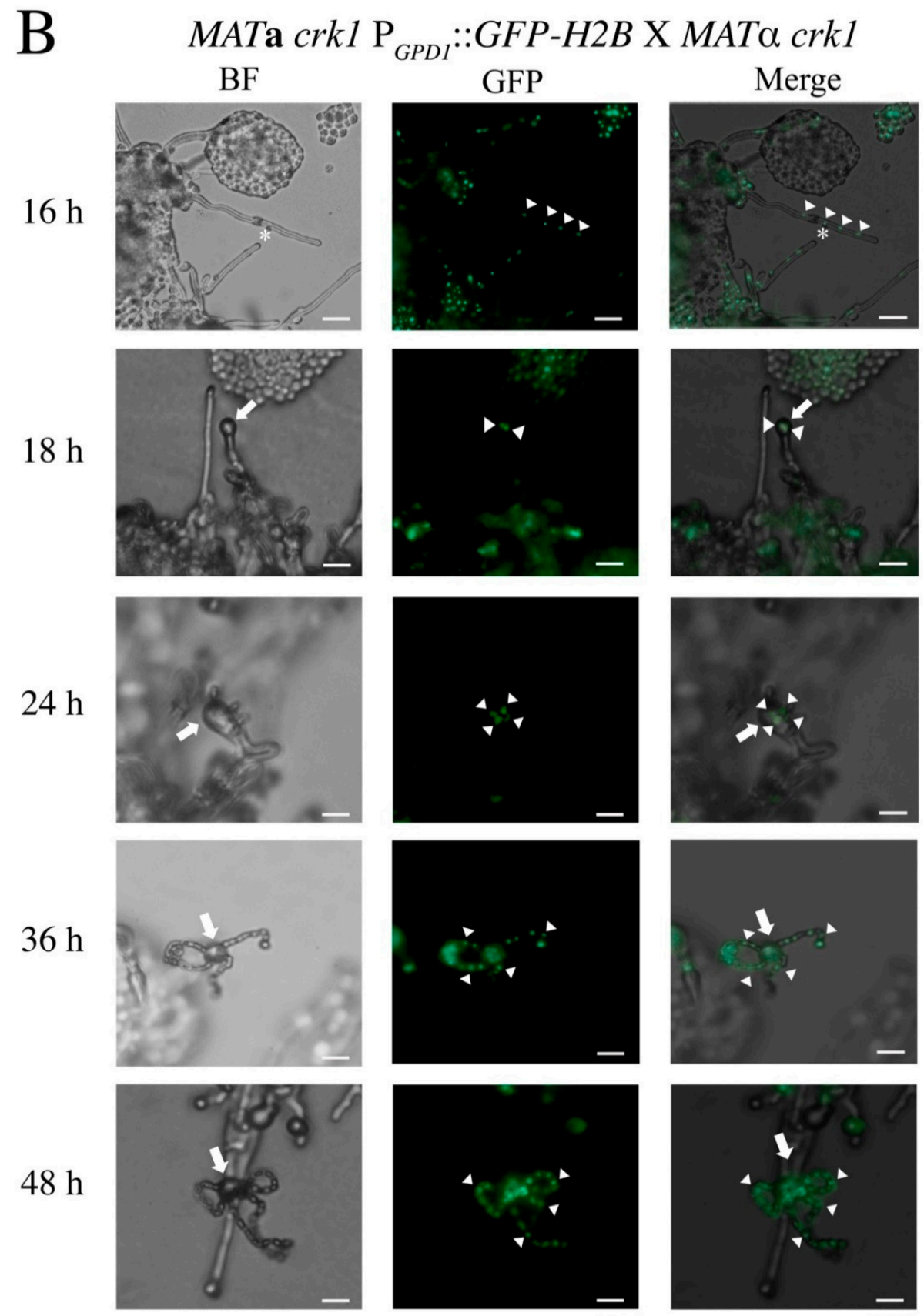

$72 \mathrm{~h}$
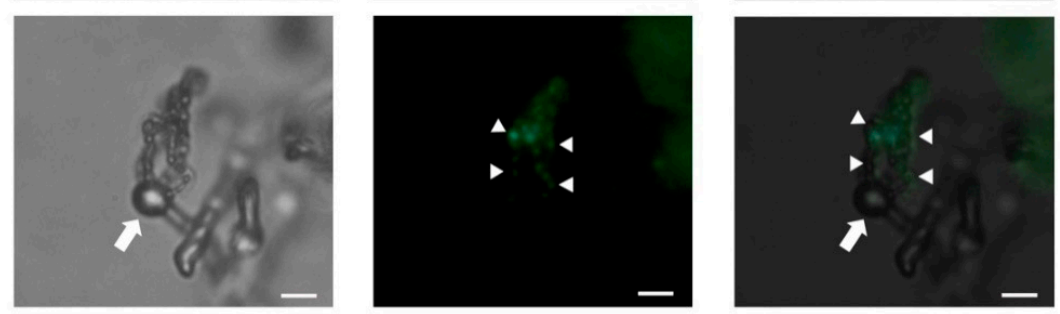

Figure 1. Nuclear distribution in the mating structures of the wild-type and bilateral crk1 mutant crosses during bisexual mating process. Mating of the wild-type (A) and bilateral crk1 mutant (B) crosses was conducted on V8 solid medium and incubated at $26^{\circ} \mathrm{C}$. Nuclei were visualized by Gfp-H2b fusion protein in the MATa wild-type and MATa crk1 mutant cells. Bright-field (BF) and fluorescent (GFP) images of dikaryotic filaments, basidia and long chains of basidiospores were photographed at $400 \times$ magnification. Photos taken from 16 to $72 \mathrm{~h}$ were shown and merged photos were created with ImageJ. White star indicates clamp cell, white triangle indicates nucleus, and white arrow indicates basidia. Scale bar $=5 \mu \mathrm{m}$. 
A

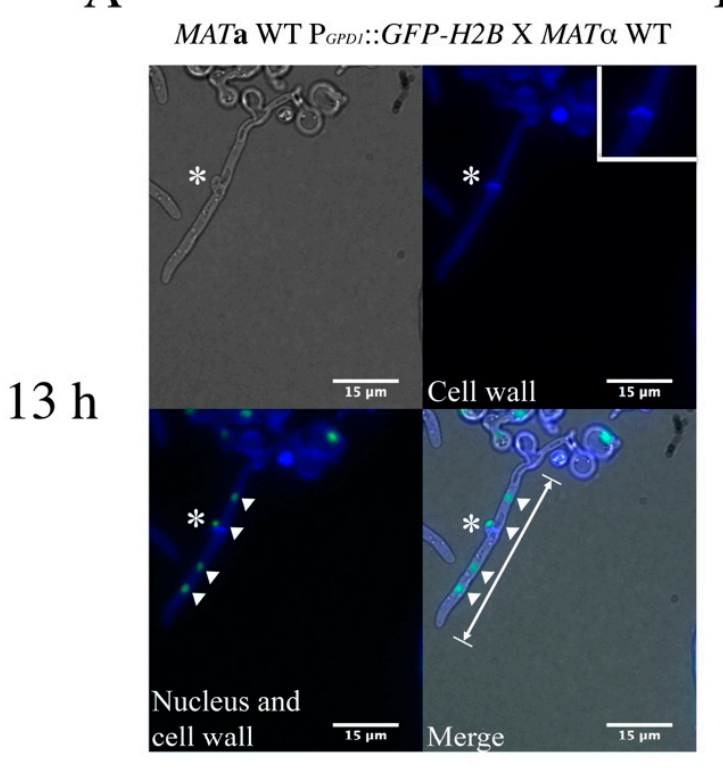

$17 \mathrm{~h}$

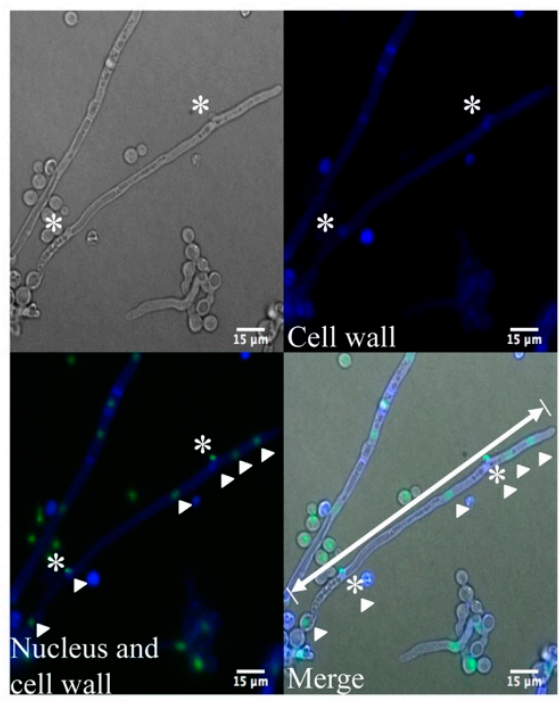

B
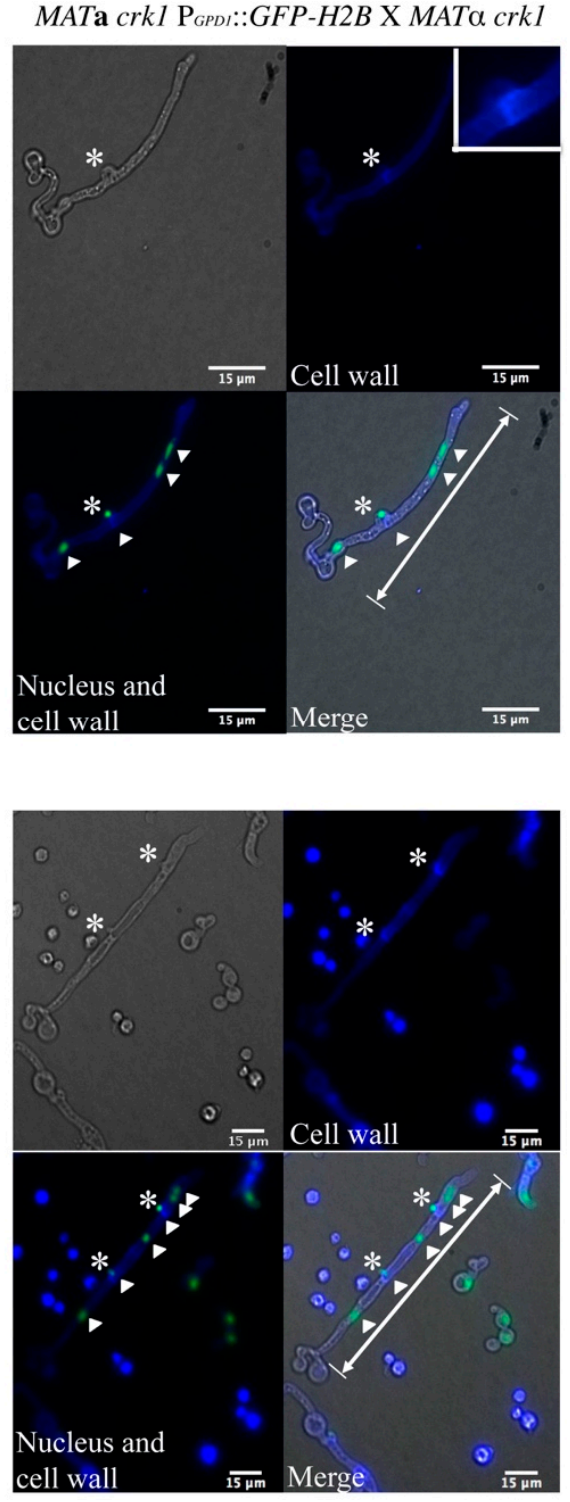

Figure 2. Morphology of dikaryotic filaments in the wild-type and bilateral crk1 mutant crosses. Mating mixtures of the wild-type (A) and bilateral crk1 mutant (B) crosses were incubated on SLAD medium containing $25 \mu \mathrm{g} / \mathrm{mL}$ calcofluor white and kept at $26^{\circ} \mathrm{C}$ under dark condition. Nuclei were labeled by Gfp-H2b fusion protein. Cell wall was stained with calcofluor white. Dikaryotic filaments were photographed at 13 and $17 \mathrm{~h}$ post-incubation at $60 \times$ magnification. White star indicates clamp cell and white triangle marks nucleus. White double-headed arrow indicates the range of dikaryotic filament measurement. Scale bar $=15 \mu \mathrm{m}$.

3.2. C. neoformans CRK1 Repressed Cell Fusion, Filamentation, Karyogamy and Meiotic Gene Expression during Bisexual Mating

The Cpk1 MAPK cascade is one of the key signaling pathways that govern pheromone transduction, cell fusion, and dikaryotic filament emergence in C. neoformans. To determine the effect of $C$. neoformans CRK1 mutation on the expression of the genes involved in the Cpk1 MAPK signaling pathway, dikaryotic filamentation, and fruiting structure development, we conducted gene expression analyses in the wild-type and crk1 mutant. Because we determined the morphological features of the wild-type and crk1 mutant in dikaryotic filamentation from $16 \mathrm{~h}$ to $72 \mathrm{~h}$ during bisexual mating, mating samples at $0,12,14,16,18,20,22$, and 24 h post-incubation on V8 medium were selected for gene expression 
study and analyzed by real-time qRT-PCR. In the wild-type, CRK1 expression was maintained at low levels from 12 to $24 \mathrm{~h}$, and the lowest mean mRNA expression for CRK1 was $0.66 \pm 0.11$-fold at $12 \mathrm{~h}$ but gradually increased and peaked at $1.62 \pm 0.04$-fold at $18 \mathrm{~h}$ (Figure $3 \mathrm{~A}$ ).

A

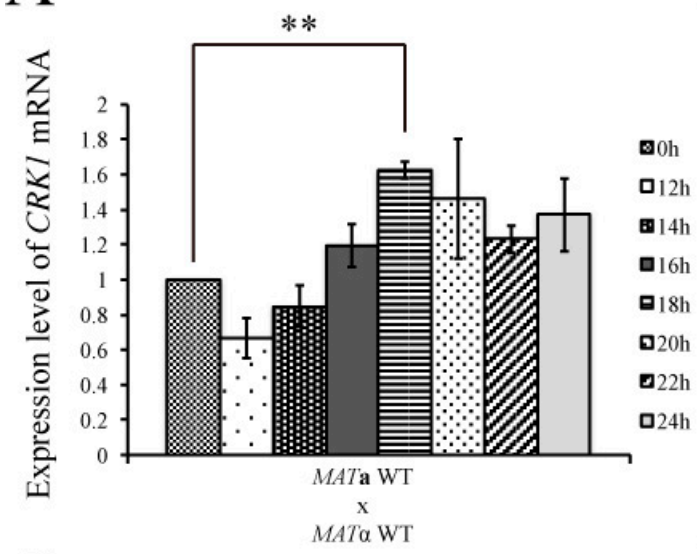

$\mathrm{C}$

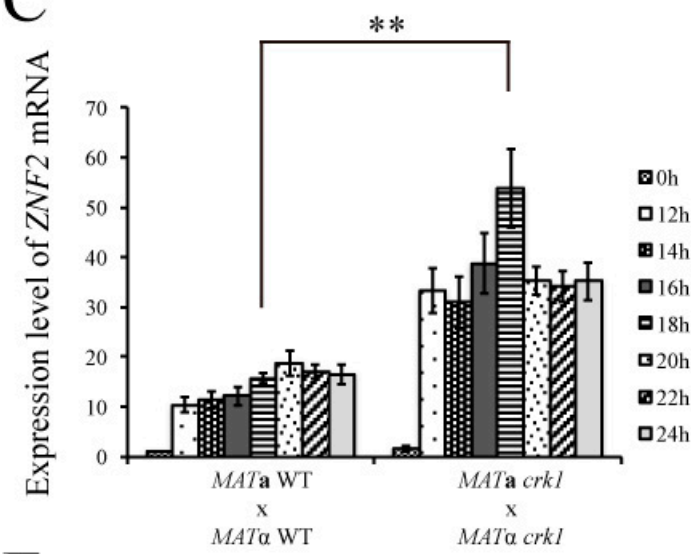

$\mathrm{E}$

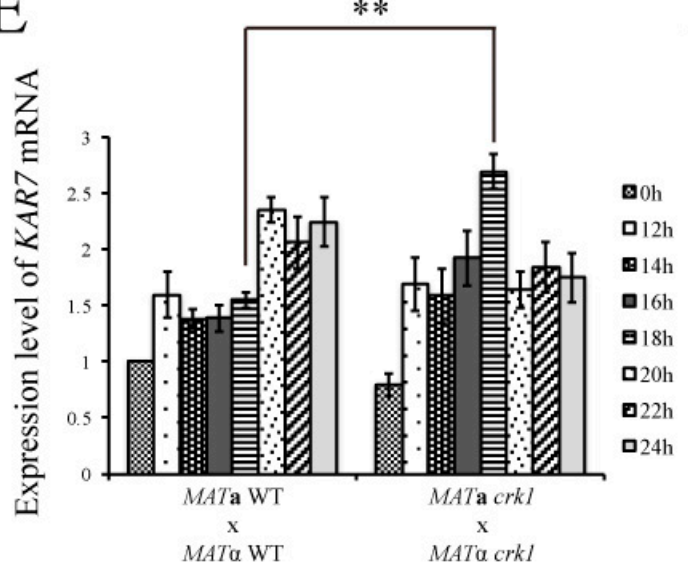

$\mathrm{B}$

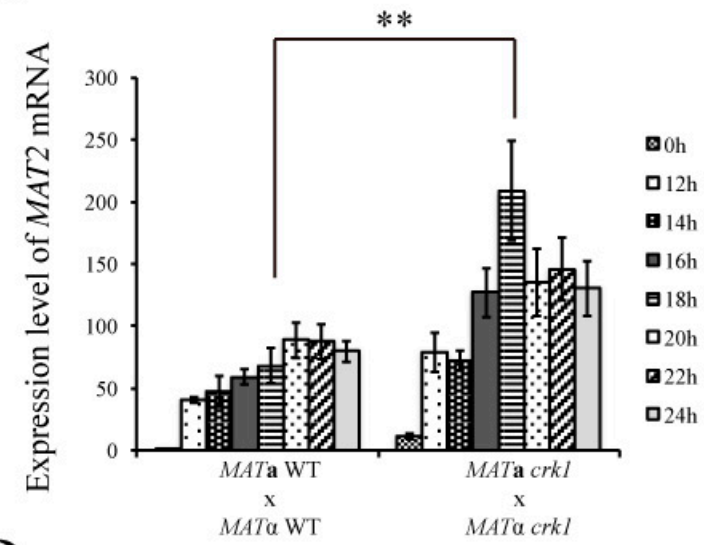

$\mathrm{D}$

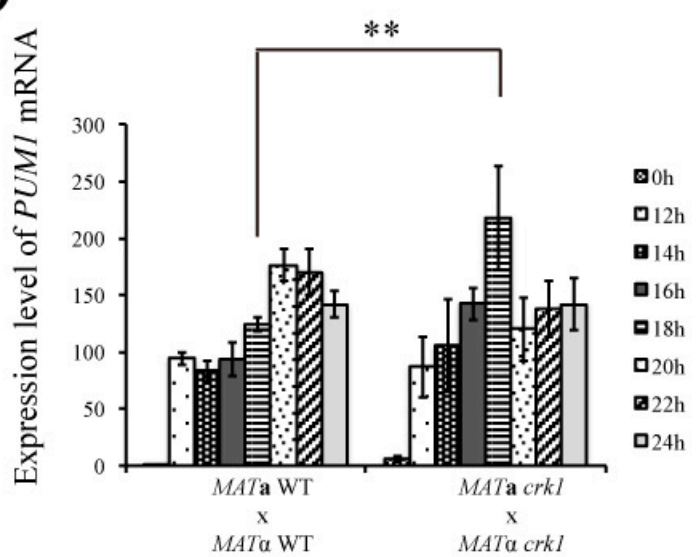

$\mathrm{F}$

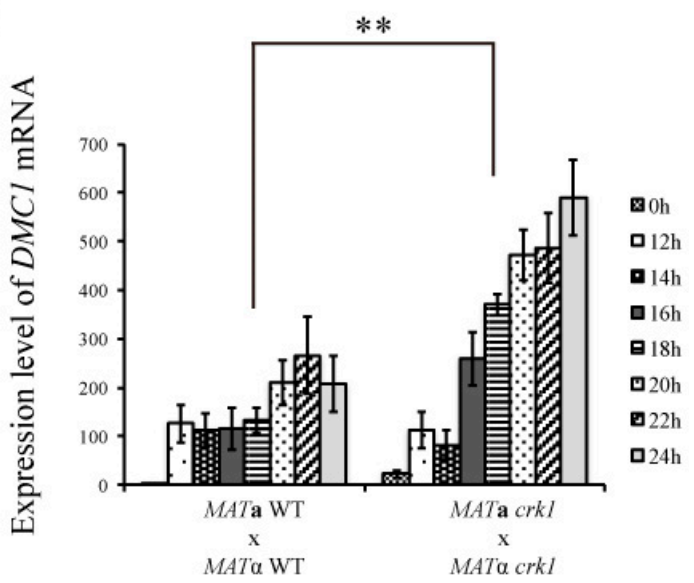

Figure 3. The expression of mating-, hyphal extension-and sporulation-related genes was elevated at $18 \mathrm{~h}$ in the bilateral $c r k 1$ mutant cross. Bilateral crosses involved the MATa and MAT $\alpha$ wild-type and $c r k 1$ mutants were conducted on V8 agar plates and incubated at $26^{\circ} \mathrm{C}$ in the dark. Samples were collected at $0,12,14,16,18,20,22$ and $24 \mathrm{~h}$ post-incubation. The expression of (A) CRK1, (B) MAT2, (C) ZNF2, (D) PUM1, (E) KAR7, and (F) DMC1 was examined by real-time qRT-PCR analysis. Triplicate reactions for each sample were conducted. Error bar represents the standard deviation from the mean of three replicates. The results were normalized to C. neoformans GPD1 expression. (** indicates $p<0.005)$. 
C. neoformans MAT2 gene is a major transcription factor that regulates pheromone production and stimulates cell fusion [16]. We next examined the MAT2 expression pattern in the wild-type and bilateral crk1 mutant crosses. The MAT2 expression was higher in the bilateral crk1 mutant cross than the wild-type from 12 to $24 \mathrm{~h}$. The mean level of MAT2 in the wild-type was $48.49 \pm 3.9$-fold at $12 \mathrm{~h}$, then peaked at $88.58 \pm 13.7$-fold at $20 \mathrm{~h}$. However, the mean mRNA level of MAT2 in the bilateral crk1 mutant cross was $79.03 \pm 16.02$-fold at $12 \mathrm{~h}$ and the peak expression was $208 \pm 39.69$-fold at $18 \mathrm{~h}$. After $18 \mathrm{~h}$, the mRNA level of MAT2 decreased (Figure 3B).

C. neoformans ZNF2 gene is the major transcription factor that regulates filament development and also induces PUM1 to generate aerial hyphae during bisexual mating [21]. ZNF2 expression was upregulated in the bilateral crk1 mutant cross as compared with the wild-type, with peak level at $18 \mathrm{~h}$ (Figure 3C). In contrast, the level of ZNF2 peaked at $18.86 \pm 2.52$-fold at $20 \mathrm{~h}$ in the wild-type but remained significantly lower than that for the bilateral crk1 mutant cross (Figure 3C). The wild-type and bilateral crk1 mutant cross did not differ in the level of PUM1 at $12 \mathrm{~h}$ and $14 \mathrm{~h}$ (Figure 3D). At 16 and $18 \mathrm{~h}$, PUM1 expression increased significantly in the bilateral crk1 mutant cross and peaked at $18 \mathrm{~h}$. The peak expression for PUM1 in the wild-type was at $20 \mathrm{~h}$ (Figure 3D). Furthermore, both ZNF2 and PUM1 expression was downregulated in the bilateral crk1 mutant cross after $18 \mathrm{~h}$ (Figure 3C,D).

We also assessed the transcript levels of karyogamy-and meiotic-related genes KAR7 and DMC1. In the wild-type, the level of KAR7 was stable from 12 to $18 \mathrm{~h}$, then increased at $20 \mathrm{~h}$. In the bilateral crk1 mutant cross, the expression level of KAR7 was elevated earlier than the wild-type at 16 and $18 \mathrm{~h}$ but decreased from 20 to $24 \mathrm{~h}$ (Figure 3E). The expression of DMC1 in the wild-type initially showed no significant changes from 12 to $18 \mathrm{~h}$ but increased at $20 \mathrm{~h}$ and peaked at $22 \mathrm{~h}$ (Figure $3 \mathrm{~F}$ ). In the bilateral crk1 mutant cross, DMC1 expression was similar to that of the wild- type at 12 and $14 \mathrm{~h}$. However, the expression markedly increased at $16 \mathrm{~h}$ and continuously increased until $24 \mathrm{~h}$ (Figure 3F). Hence, our gene expression results showed that the mutation of CRK1 resulted in upregulation of cell fusion-, filamentation-, karyogamy-and meiotic-related genes at about 16 to $18 \mathrm{~h}$ during bisexual mating, indicating C. neoformans CRK1 played important roles in regulating various bisexual differentiation processes.

\subsection{CRK1 Expression Level was Decreased in the Bilateral mat2 Mutant Cross}

In this research, we confirm that MAT2 expression was increased in the bilateral crk1 mutant cross. Our previous study also showed that $C R K 1$ overexpression downregulates the transcript level of MAT2 during bisexual mating [29]. To further assess the relation between CRK1 and MAT2, we generated the crk1mat2 double mutant for phenotypic and gene expression analyses. Unilateral and bilateral crosses of the mat2, crk1, and crk1mat2 mutant strains were examined on V8 medium for $24 \mathrm{~h}$. Dikaryotic filaments were observed in the bilateral crk1 mutant cross but were blocked under mat2 deletion (Figure 4A). Both unilateral and bilateral crk1mat2 double mutants did not produce dikaryotic filaments, even when the crk1mat2 double mutant was crossed with the crk1 mutant strain. Furthermore, we examined CRK1 and MAT2 expression in the wild-type and crk1, mat2 and crk1mat2 double mutant during bisexual mating on V8 medium at $0,6,18$ and $24 \mathrm{~h}$. MAT2 mRNA level was not detected in the bilateral mat 2 or crk1mat 2 double mutant cross, and CRK1 was not detected in the bilateral crk1 or crk1mat 2 double mutant cross. In the bilateral crk1 mutant cross, the level of MAT2 was similar to that in the wild-type at $6 \mathrm{~h}$ but then became higher than the wild-type starting at $12 \mathrm{~h}$ (Figure 4B), as also demonstrated in Figure 3B. Interestingly, the level of CRK1 was downregulated in the bilateral mat 2 mutant cross (Figure $4 \mathrm{C}$ ). 
A

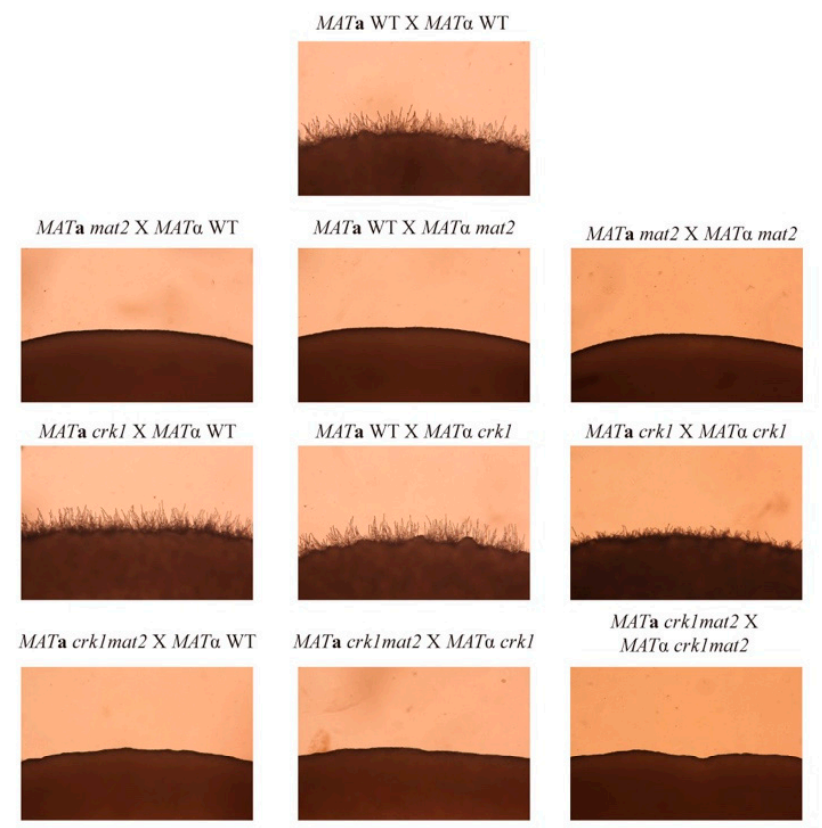

B

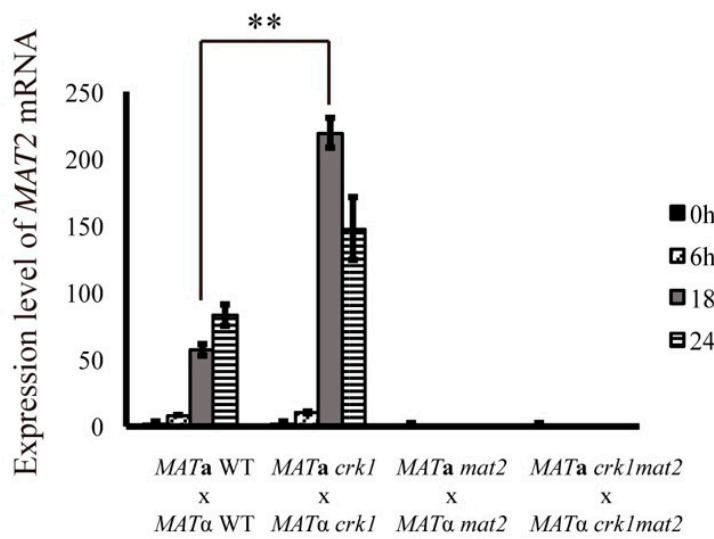

$\mathrm{C}$

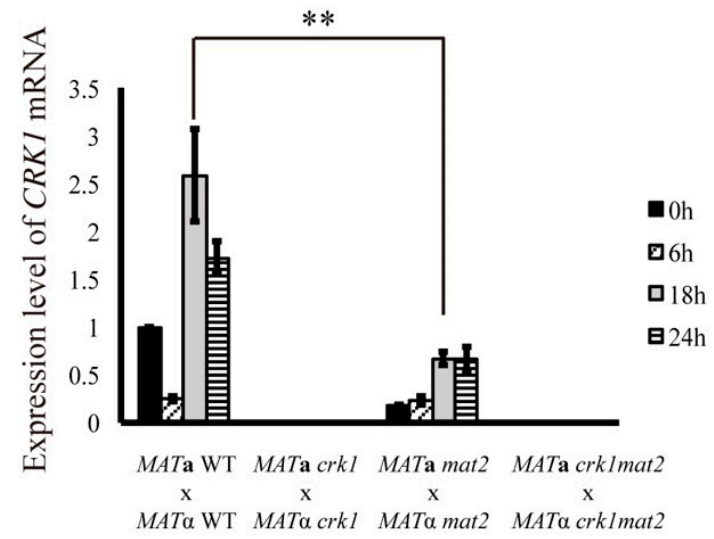

Figure 4. Sexual differentiation in the crk1 mutant was blocked by the mutation of MAT2 and CRK1 expression was reduced in the bilateral mat 2 mutant cross. (A) C. neoformans MATa and MAT $\alpha$ strains were crossed as indicated. Mating was conducted on V8 agar plates and incubated at $26{ }^{\circ} \mathrm{C}$ in the dark. Photos were taken $24 \mathrm{~h}$ post-incubation at $100 \times$ magnification. Bilateral crosses involved the MATa and $M A T \alpha$ wild-type and $c r k 1$, mat2, and mat2crk1 double mutants were conducted. Samples were collected at $0,6,18$ and 24 h post-incubation and subjected to RNA extraction. The expression of MAT2 (B) and CRK1 (C) during bisexual mating was examined by real-time qRT-PCR analysis. Triplicate reactions for each sample were conducted. Error bar represents the standard deviation from the mean of three replicates. The results were normalized to C. neoformans GPD1 expression. (** indicates $p<0.005$ ).

Because the mRNA level of CRK1 was decreased in the bilateral mat2 mutant cross, we further assessed the effect of MAT2 overexpression on the CRK1 level during bisexual mating. MAT2 overexpression was constructed in the C. neoformans MAT $\alpha$ strain, and bisexual mating was conducted on V8 medium for $24 \mathrm{~h}$. Profuse filaments were observed around the edge of mating colonies in the $M A T \alpha \mathrm{P}_{G P D 1}:: M A T 2$ strain cross in comparison with the wild-type cross (Figure 5A). The mRNA level of $M A T 2$ was greatly elevated in the $M A T \alpha \mathrm{P}_{G P D 1}:: M A T 2$ strain (Figure $5 \mathrm{~B}$ ). The expression of $S X I 1 \alpha$, a downstream target of MAT2 [5], was also increased markedly at 0 and $24 \mathrm{~h}$ and was significantly 
higher than in the wild-type (Figure 5C). The mRNA level of CRK1 at 0 and $24 \mathrm{~h}$ was higher than in the wild-type (Figure 5D). Thus, the deletion of MAT2 blocked dikaryotic filamentation of the crk1 mutants during the bisexual mating process. However, C. neoformans MAT2 likely regulated CRK1 at the transcription level.

A
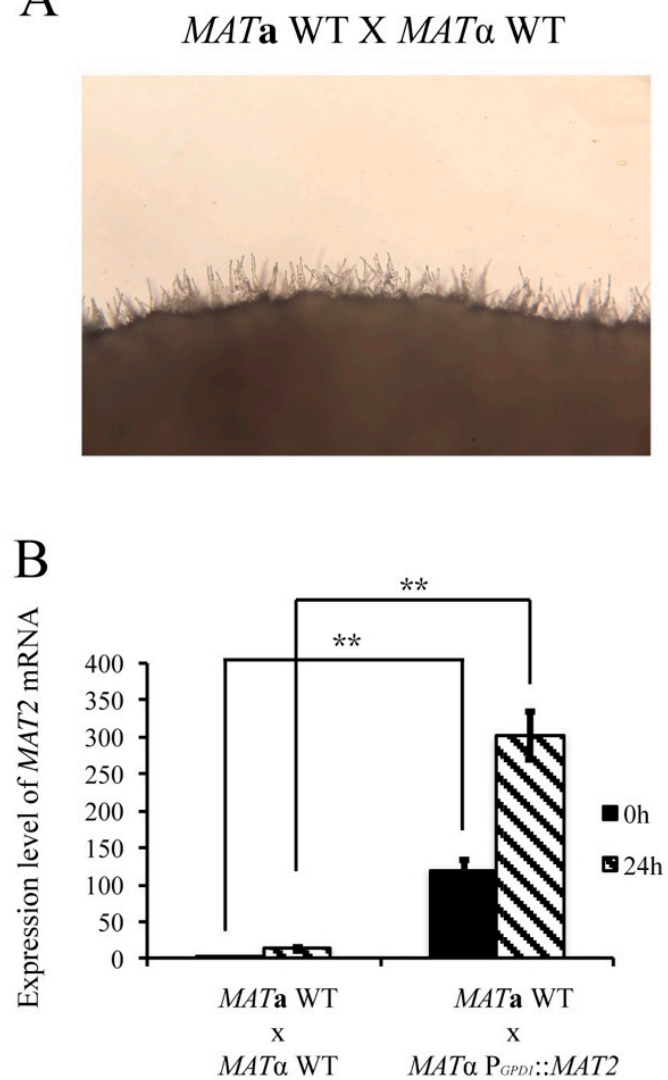

MATa WT X MAT $\alpha \mathrm{P}_{G P D I:: M A T 2}$
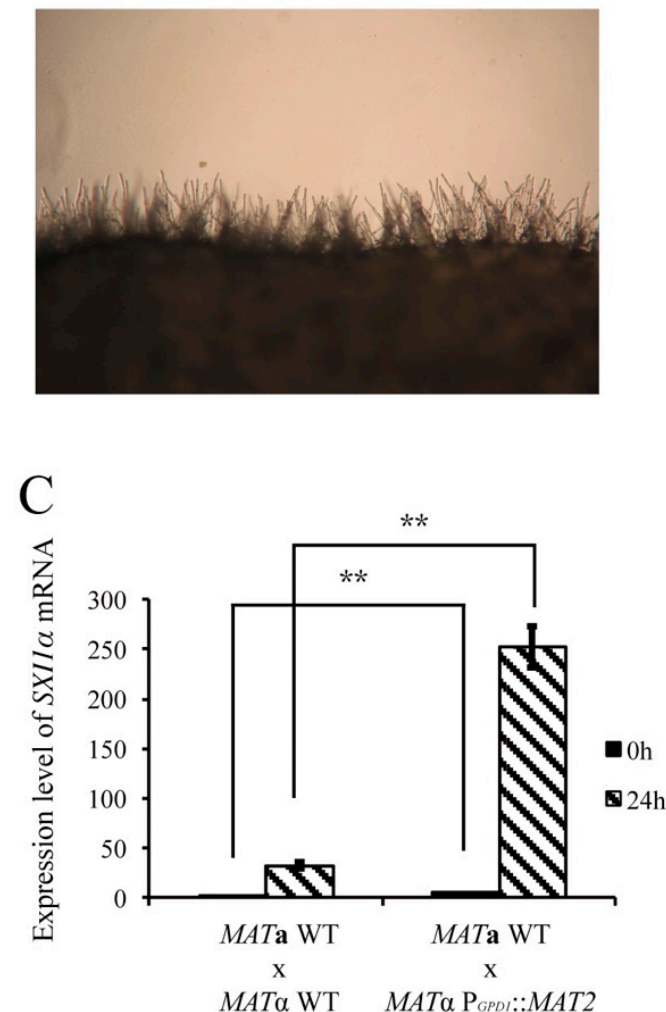

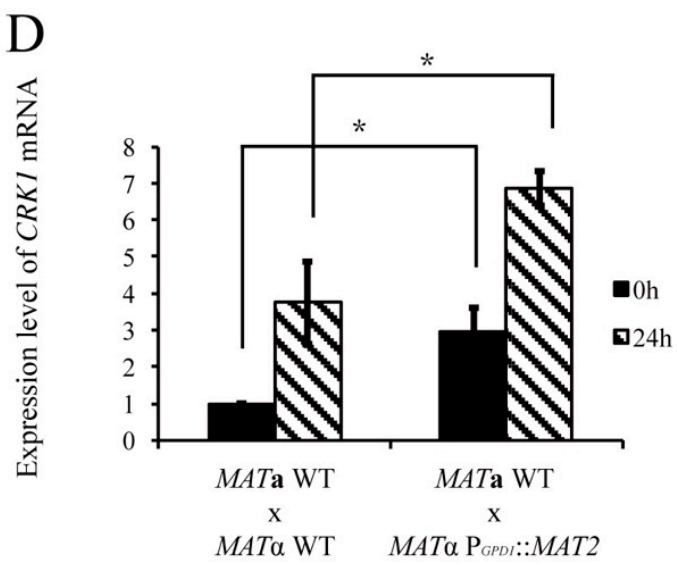

Figure 5. Overexpression of MAT2 increased bisexual filamentation and upregulated CRK1 expression. (A) Crosses involved C. neoformans MATa and MAT $\alpha$ wild-type and MATa wild-type and MAT $\alpha$ $\mathrm{P}_{G P D 1}:: M A T 2$ strains were conducted on V8 agar plates incubated at $26{ }^{\circ} \mathrm{C}$ in the dark. Photos were taken $24 \mathrm{~h}$ post-incubation at $100 \times$ magnification. The same crosses were also subjected to gene expression studies and samples were collected at 0 and $24 \mathrm{~h}$ post-incubation. The expression of MAT2 (B), SXI1 $\alpha(\mathbf{C})$, and CRK1 (D) during mating was determined by real-time qRT-PCR analysis. Triplicate reactions for each sample were conducted. Error bar represents the standard deviation from the mean of three replicates. The results were normalized to C. neoformans GPD1 expression. (* indicates $p<0.005$; ${ }^{*}$ indicates $\left.p<0.05\right)$. 


\subsection{Deletion of C. neoformans ZNF2 Resulted in Non-Filamentation in the crk1 Mutant}

C. neoformans ZNF2 is a positive regulator for filament generation; deletion of ZNF2 abolished the formation of dikaryotic filaments [16,19]. Previously, the expression of ZNF2 was found negatively regulated by CRK1 during bisexual mating. To assess whether crk1 deletion could restore filamentation in the $z n f 2$ mutant during bisexual differentiation, we generated the $c r k 1 z n f 2$ double mutant to examine the bisexual mating phenotype and compared with the wild-type, crk1 and znf2 crosses. As previously described, abundant filaments were seen in the $c r k 1$ cross, and filamentation was blocked in the bilateral $z n f 2$ mutant cross. Similarly, no dikaryotic filaments were observed in the bilateral crk1znf2 mutant cross and ZNF2 deletion blocked the filamentation phenotype of znf2 mutant (Figure S2A).

To further determine the relation between CRK1 and ZNF2, we examined the expression of CRK1 in the wild-type and $z n f 2$ deletion strains during bisexual mating on V8 medium for 6, 18 and $24 \mathrm{~h}$. $M F \alpha$ pheromone gene is a positive indicator for bisexual mating process. The $M F \alpha$ expression was upregulated, peaked at $18 \mathrm{~h}$ in the $z n f 2$ cross and then decreased at $24 \mathrm{~h}$. In contrast, the expression of $M F \alpha$ was lower in the wild-type than the $z n f 2$ cross (Supplementary Figure S2B) as previously described [16]. The mRNA levels of CRK1 did not differ between the wild-type and znf2 cross (Supplementary Figure S2C). Our results suggested that CRK1 was not regulated by ZNF2, and deletion of the negative regulator CRK1 did not restore filamentation in the znf2 mutant background.

\subsection{Gat1 Contains the Predicted Crk1 Consensus Phosphorylation Site}

C. neoformans CRK1 is a homologue of S. cerevisiae IME2 gene. The IME2 phosphoacceptor consensus sequence R-P-X-S/T-R/P/A was characterized and predicted in several fungal species, including N. crassa and $C$. neoformans $[35,42,43]$. To predict the putative phosphorylation targets of C. neoformans Crk1, we used a bioinformatic strategy to search the $C$. neoformans JEC21 database for proteins that contain the modified S. cerevisiae Ime2 phosphorylation consensus sequence R-P-X-S/T-R/P/A. Overall, 651 putative phosphorylation targets of Crk1 were predicted from the C. neoformans JEC21 genome and proteins containing DNA binding motif were further selected. From the C. neoformans JEC21 transcription factor database, 22 genes predicted as transcription factors were further identified, and these homologous genes in C. neoformans H99 were identified (Supplementary Table S2). Nitrogen starvation is one of the conditions that induces bisexual mating in C. neoformans [4]. The GATA type transcription activator Gat1 was predicted as a putative Crk1 phosphorylated substrate in our bioinformatic survey. C. neoformans GAT1 is homologous to N. crassa nit2 and Schizosaccharomyces pombe gaf1, the regulators of nitrogen metabolism [55-57]. C. neoformans Gat1 contains the GATA zinc-finger domain at the C-terminus (1186 to $1230 \mathrm{aa}$ ), and the putative IME2 phosphorylation consensus sequence was predicted at RPGT $^{* 1164}$. Previous studies indicated that GAT1 has a negative function for bisexual mating in C. neoformans H99 [58,59]. We further tested its function for bisexual differentiation in C. neoformans JEC21 and a genetic association with C. neoformans CRK1.

\subsection{Deletion of GAT1 Enhanced Pheromone Expression and Increased Aerial Hyphae Formation}

To dissect the function of GAT1 in bisexual mating, we generated gat1 deletion strains by double-joint PCR in both MATa and MAT $\alpha$ wild-type strains. Complementation of the mutant strains with the wild-type GAT1 gene was also constructed. Bisexual mating assay for the unilateral and bilateral gat1 mutant crosses was conducted on V8 medium in the dark. After 24-h incubation, the abundance of dikaryotic filaments around the edge of mating mixture was slightly enhanced in the unilateral gat 1 cross and more profuse filaments were obviously seen in the bilateral gat1 cross as compared with the wild-type cross (Figure 6A). Increased filamentation was similarly observed in the bilateral crk1 mutant cross, however, filaments were longer in the bilateral gat1 mutant cross than bilateral crk1 mutant cross. Furthermore, profuse aerial hyphae were generated in the bilateral gat 1 mutant cross on the surface of mating mixture, which was similar to the bilateral crk1 mutant cross (Figure 6B). 
A
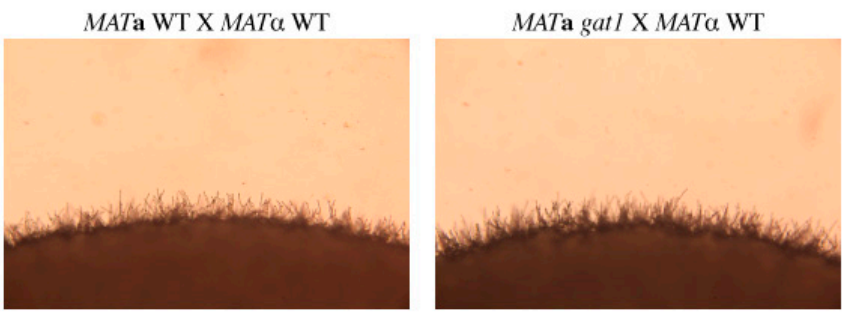

MATa WT X MATa gat l

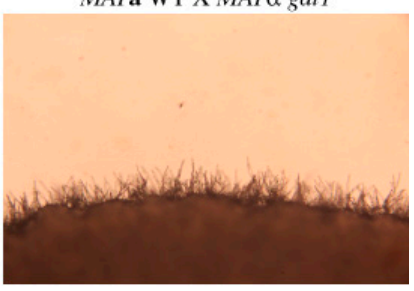

MATa gatl X MATa gatl
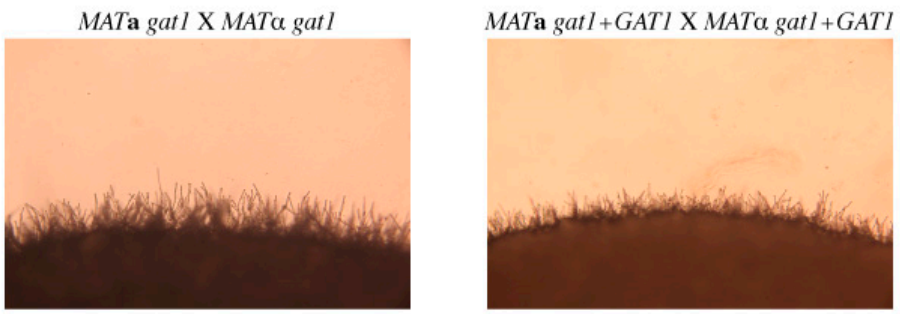

B

MATa WT X MAT $\alpha$ WT

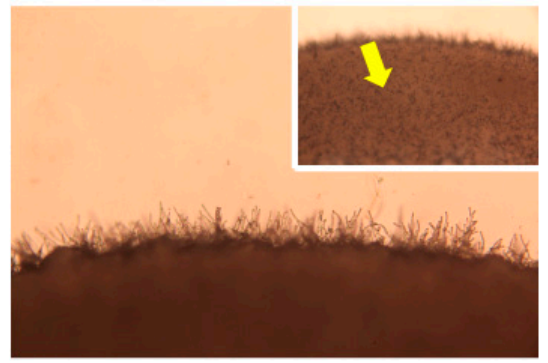

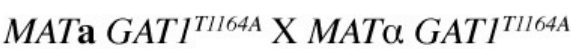

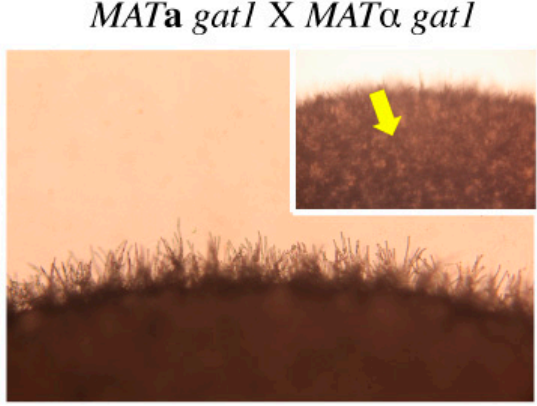

MATa $c r k l$ X MAT $\alpha$ crkl
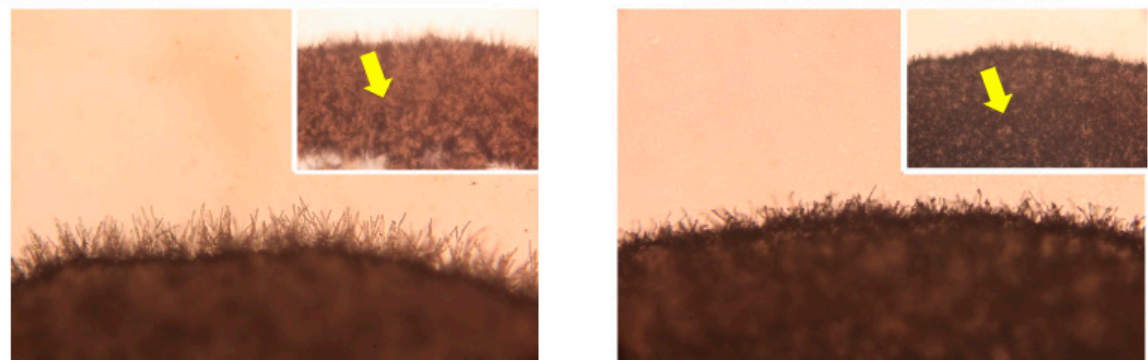

Figure 6. Dikaryotic filamentation and aerial hyphae formation were enhanced in the bilateral gat1 mutant and GAT1 ${ }^{\mathrm{T} 1164 \mathrm{~A}}$ mutant crosses. (A) C. neoformans MATa and MAT $\alpha$ gat1 mutant and overexpression strains were crossed as indicated and compared to the wild type cross. (B) C. neoformans MATa and MAT $\alpha$ GAT1 ${ }^{\mathrm{T} 1164 \mathrm{~A}}$ bilateral mutant cross was performed and compared to the crosses as indicated. Small photos reveal the density of aerial hyphae. Yellow arrow indicates aerial hyphae. Mating was conducted on V8 agar plates and incubated at $26{ }^{\circ} \mathrm{C}$ in the dark. Photos were taken $24 \mathrm{~h}$ post-incubation at $100 \times$ magnification.

To investigate how C. neoformans GAT1 gene is involved in the signaling networks of mating process, the wild-type and gat1 bilateral mutant crosses were conducted on V8 medium, and mating cells were harvested at 12,14,16, 18, 20, 22, and $24 \mathrm{~h}$ for gene expression studies by real-time qRT-PCR. The expression of GAT1 in the wild-type cross was $3.88 \pm 0.12$-fold at $12 \mathrm{~h}$ and reached to the highest expression level at $20 \mathrm{~h}$ (Figure 7A). The mRNA level of $M F \alpha$ in the wild-type cross was elevated during mating process, peaked at $18 \mathrm{~h}$ and then decreased afterwards (Figure $7 \mathrm{~B}$ ). $M F \alpha$ expression was increased greatly since $12 \mathrm{~h}$ in the bilateral gat 1 mutant cross and maintained at high levels till $24 \mathrm{~h}$. In addition, we further analyzed the mRNA levels of MAT2 and PUM1 to correlate the pheromone expression and aerial hyphae formation. Interestingly, the expression levels of MAT2 between the 
wild-type and bilateral gat 1 mutant cross were similar at 12 and $14 \mathrm{~h}$, but were increased since $16 \mathrm{~h}$ in the bilateral gat1 mutant cross (Figure 7C). The expression of PUM1 was generally increased in the bilateral gat1 mutant cross as compared with the wild-type cross and significantly up-regulated at $24 \mathrm{~h}$ (Figure 7D). Thus, according to phenotypic and expression analysis, C. neoformans GAT1 also played a negative role in the regulation of mating-related genes and deletion of GAT1 resulted in abundant aerial hyphae formation.
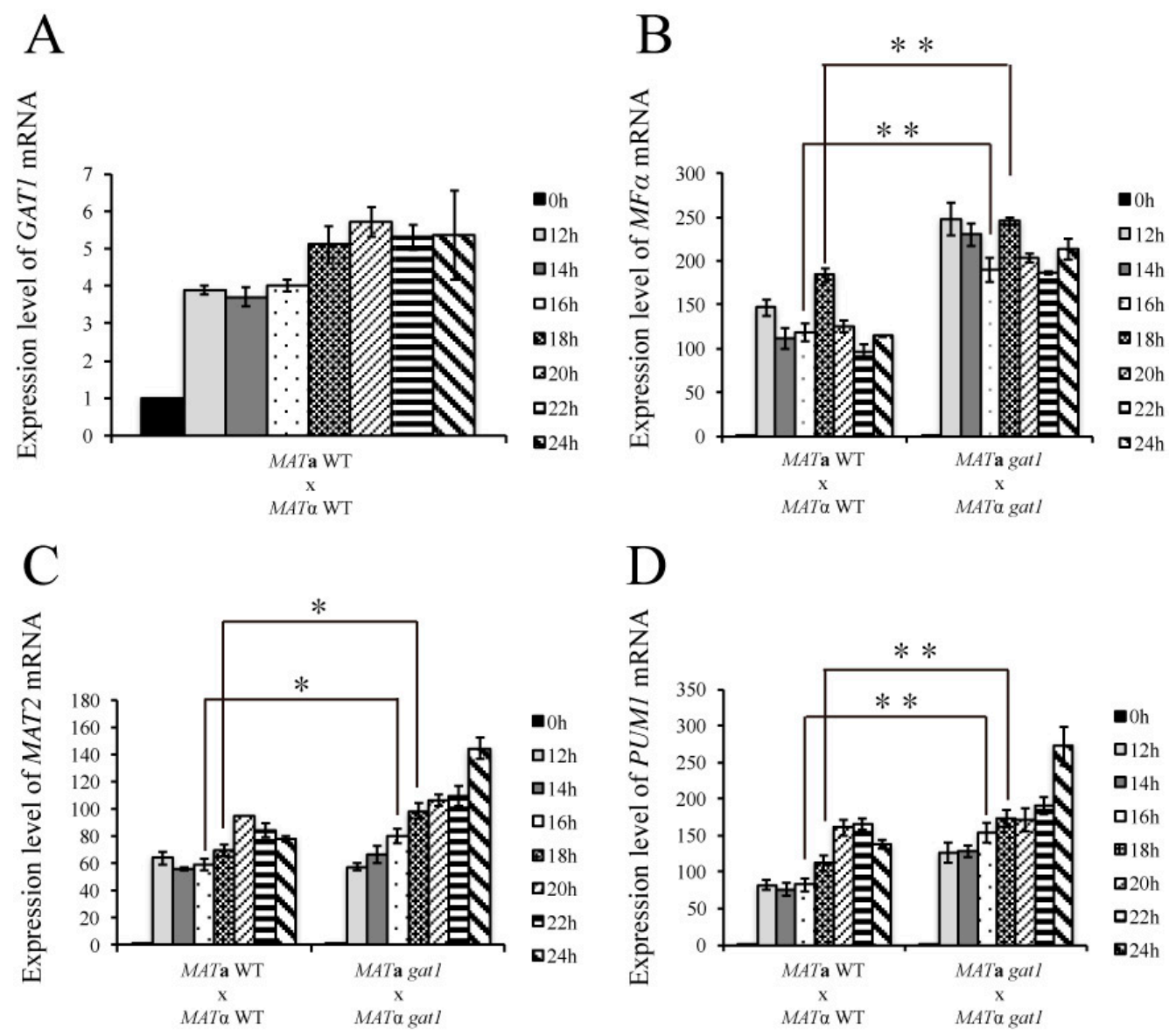

Figure 7. The expression of mating- and hyphal extension-related genes was increased in the bilateral gat1 mutant cross. Bilateral crosses involved the MATa and MAT $\alpha$ wild-type and gat 1 mutants were conducted on V8 agar plates and incubated at $26^{\circ} \mathrm{C}$ in the dark. Samples were collected at $0,12,14,16$, 18, 20, 22 and 24 h post-incubation. The expression of GAT1 (A), MF $\alpha$ (B), MAT2 (C), and PUM1 (D) was examined by real-time qRT-PCR analysis. Error bar represents the standard deviation from the mean of three replicates.Triplicate reactions for each sample were conducted. The results were normalized to C. neoformans GPD1 expression. (** indicates $p<0.005 ;{ }^{*}$ indicates $p<0.05$ ).

\subsection{The C. neoformans crk1gat1 Mutants Exhibited Similar Phenotypes as the crk1 Mutant in Bisexual Mating}

As we suggested before that Crk1 may phosphorylate Gat1 to regulate bisexual mating in C. neoformans, we further generated the crk1gat1 mutants to further dissect the genetic relationship between CRK1 and GAT1 in bisexual mating. The bilateral crk1gat1 mutant cross were conducted on V8 medium in the dark. After $24 \mathrm{~h}$ incubation, short dikaryotic filaments at the edge of mating colonies were observed as the bilateral crk1 mutant cross and basidia also emerged both in the bilateral crk1 and crk1gat1 mutant cross (Figure 8A,B). We also further examined the expression levels of $M F \alpha$ and MAT2 genes. The wild-type, bilateral gat1 mutant, crk1 mutant and crk1gat1 mutant crosses were incubated on V8 medium and mating cells were harvested at 18 and $24 \mathrm{~h}$ for real-time qRT-PCR analyses. As shown in Supplementary Figure S3A,B, MF $\alpha$ and MAT2 expression levels in the bilateral crk1 and crk1gat1 mutant crosses were higher than those in the wild-type and gat1 mutant crosses. 
The transcript levels of $M F \alpha$ in the bilateral crk1gat1 mutant cross were higher than those of the crk1 mutant cross both at 18 and $24 \mathrm{~h}$. The transcript levels of MAT2 at $18 \mathrm{~h}$ in the bilateral crk1 and crk1gat1 mutant crosses were higher than those of the wild-type and bilateral crk1 mutant crosses. MAT2 expression level in the bilateral crk1gat1 mutant cross was also higher in comparison to the bilateral crk1 mutant cross. The C. neoformans CSA1 gene is a key regulator for basidial formation [25], and DMC1 is responsible for meiotic process. As expected, these two genes were expressed at the similar levels in the bilateral crk1 and crk1gat1 mutant crosses at 18 and $24 \mathrm{~h}$, but significantly higher than the wild-type and bilateral gat1 mutant crosses (Supplementary Figure S3C,D). Altogether, GAT1 and CRK1 both regulated the expression of mating-related genes and they may function in the same and independent signaling pathways.

A

MATa WT x MATa WT

$16 \mathrm{~h}$

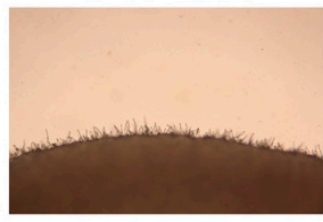

$24 \mathrm{~h}$

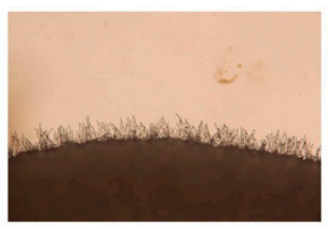

B

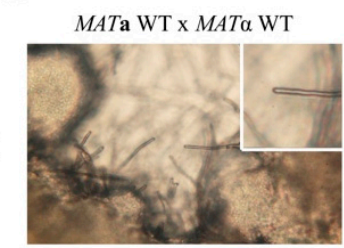

$24 \mathrm{~h}$
MATa gat1 x MATa gat1
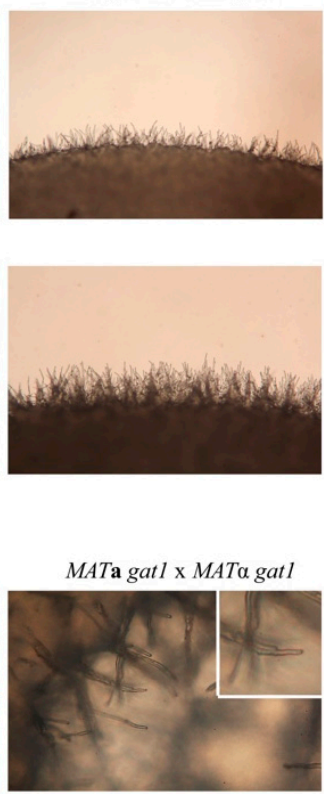

MATa crkl x MATa crkl
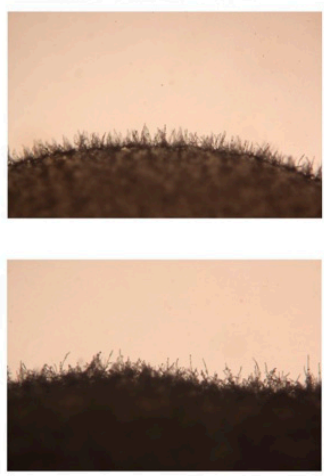

MATa crkl x MAT a crkl

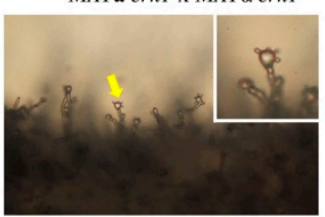

MATa crklgatl x MATa crklgat I
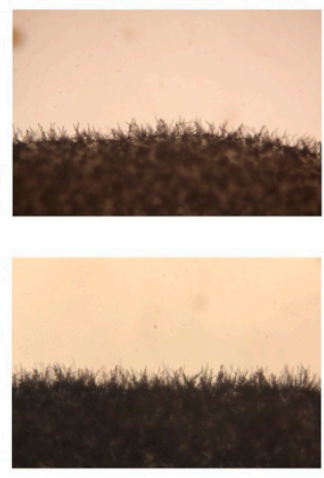

MATa crklgatl x MATa crklgat 1

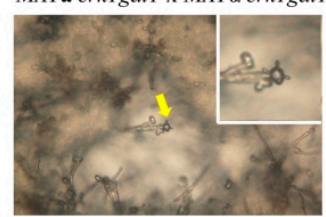

Figure 8. The $c r k 1$ and $c r k 1 g a t 1$ mutants were phenotypically identical in bisexual mating. C. neoformans MATa and MAT $\alpha$ gat1, crk1, and crk1gat 1 mutant strains were crossed on V8 agar plates at $26{ }^{\circ} \mathrm{C}$ in the dark and compared to the wild type strains. The edges of mating mixtures were photographed $16 \mathrm{~h}$ and $24 \mathrm{~h}$ post-incubation at $100 \times$ magnification. Photos of mating filaments were also recorded at $400 \times$ magnification. Small photos illustrate the tip of dikaryotic filament and yellow arrow indicates basidium.

\subsection{The Predicted IME2 Consensus Phosphorylation Site Was Important for the Function of Gat1 in} Bisexual Mating

C. neoformans Gat1 was predicted as a putative phosphorylation substrate of Crk1 and contained the Ime 2 consensus phosphorylation site RPGT ${ }^{*} 1164$. To test whether this site was important for Gat1 function to regulate bisexual mating, we conducted site-directed mutagenesis to create a gat1 phospho-null allele with the Ime 2 consensus phosphorylation site RPGT ${ }^{* 1164}$ mutated to RPGA ${ }^{* 1164}$, and also a gat1 phosphomimetic allele with RPGD ${ }^{* 1164}$. The bilateral GAT1 ${ }^{\mathrm{T} 1164 \mathrm{~A}}$ or GAT1 ${ }^{\mathrm{T} 1164 \mathrm{D}}$ mutant crosses were conducted on V8 medium for $24 \mathrm{~h}$ and compared with the wild-type and bilateral gat1 mutant crosses. In the bilateral GAT1 ${ }^{\mathrm{T} 1164 \mathrm{~A}}$ cross, the abundance of dikaryotic filaments and aerial hyphae were similar to that in the bilateral gat1 mutant cross (Figure 6B). For gene expression, GAT1 expression was not detected in the bilateral gat1 mutant cross. However, the expression levels at $24 \mathrm{~h}$ were similarly up-regulated in the wild-type, bilateral crk1 mutant cross and bilateral GAT1 ${ }^{\mathrm{T} 1164 \mathrm{~A}}$ cross (Supplementary Figure S9A). MF $\alpha$ expression was upregulated in the bilateral $c r k 1$, gat1, and GAT1 ${ }^{\mathrm{T} 1164 \mathrm{~A}}$ mutant crosses in comparison to the wild-type cross (Supplementary Figure S9B). 
On the other hand, the MATa and MAT $\alpha$ wild-type strains or crk1 mutants containing the gat1 phosphomimetic allele were used to conduct bisexual mating on V8 medium and filamentation was assessed and compared with the wild-type and bilateral crk1 mutant crosses at $16 \mathrm{~h}$ and $24 \mathrm{~h}$. In the bilateral GAT1 ${ }^{T 1164 D}$ mutant cross, dikaryotic filaments were dramatically repressed at $16 \mathrm{~h}$ and $24 \mathrm{~h}$ as compared to the wild-type cross (Figure 9A). Gene expression studies revealed that the GAT1 mRNA levels at $18 \mathrm{~h}$ and $24 \mathrm{~h}$ in the cross of GAT1 phosphomimetic strains showed no significant difference when compared to the wild-type and bilateral crk1 mutant cross (Figure 10A). In contrast, the transcript levels of $M F \alpha$ and $M A T 2$ were all significantly reduced (Figure 10B,C). In the bilateral $c r k 1+G A T 1^{\mathrm{T} 1164 \mathrm{D}}$ mutant cross, dikaryotic filaments at the edge of the mating mixture were less abundant as compared to the bilateral crk 1 mutant cross both at $16 \mathrm{~h}$ and $24 \mathrm{~h}$, but still more than wild-type cross (Figure 9A). Interestingly, the detailed features in the crk1+ GAT1 ${ }^{\mathrm{T} 1164 \mathrm{D}}$ mutant cross mimicked those in the crk1 cross. Dikaryotic filaments were shorter and basidia could be seen at $24 \mathrm{~h}$; however, the amount of basidia in the crk1+GAT1 ${ }^{\mathrm{T} 1164 \mathrm{D}}$ mutant cross was less than the bilateral crk1 cross (Figure 9B). Furthermore, the GAT1 mRNA levels at $18 \mathrm{~h}$ and $24 \mathrm{~h}$ in the cross of $c r k 1+$ GAT1 ${ }^{\mathrm{T} 1164 \mathrm{D}}$ mutants were also comparable to the levels in the wild-type and bilateral crk1 mutant cross (Figure 10A). The expression levels of $M F \alpha$ and MAT2 in the $c r k 1+G A T 1^{\mathrm{T} 1164 \mathrm{D}}$ mutants cross were reduced as compared to the crk1 mutants cross, but still maintained at high levels in comparison to the wild-type cross (Figure 10B,C). The expression levels of CSA1 for basidial formation and DMC1 for meiosis were similarly down-regulated in the $c r k 1+G A T 1^{\mathrm{T} 1164 \mathrm{D}}$ mutants cross when compared to the crk1 mutant cross (Figure 10D,E). Taken together, our results demonstrated that the Ime 2 consensus phosphorylation site of Gat1 was essential for the proper regulatory function of Gat1 in bisexual mating.
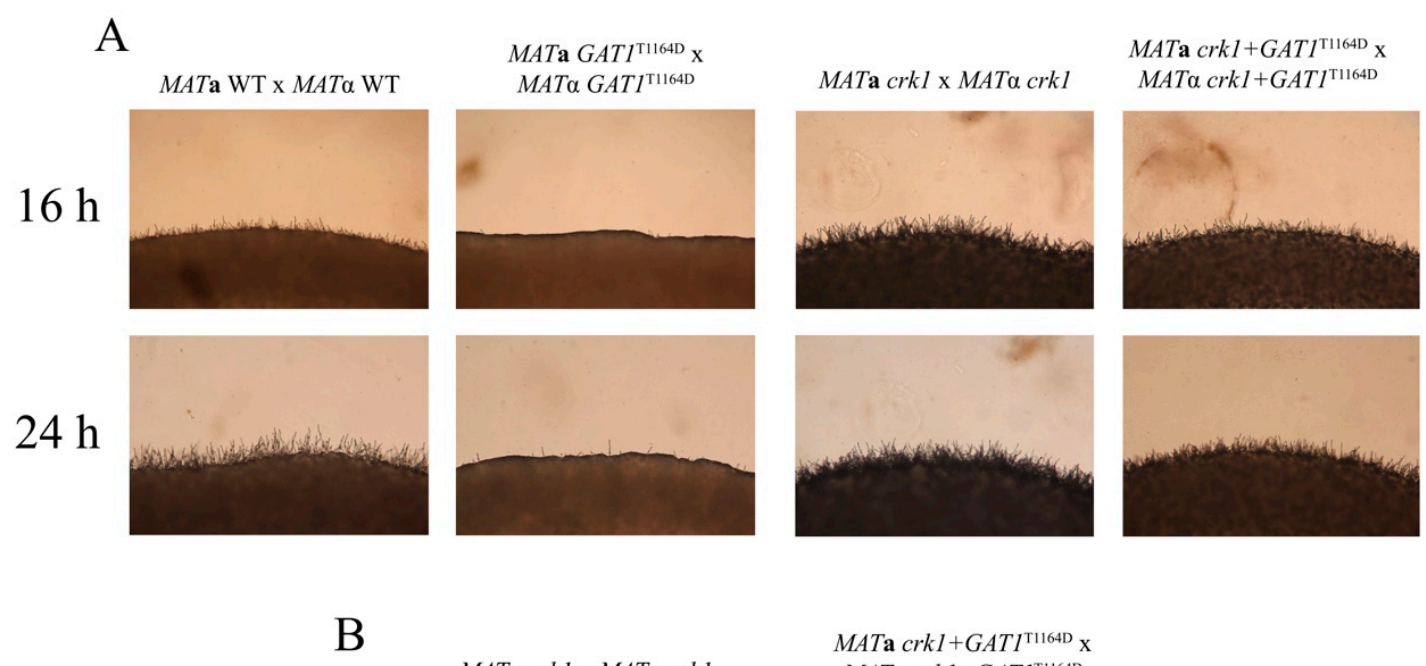

B

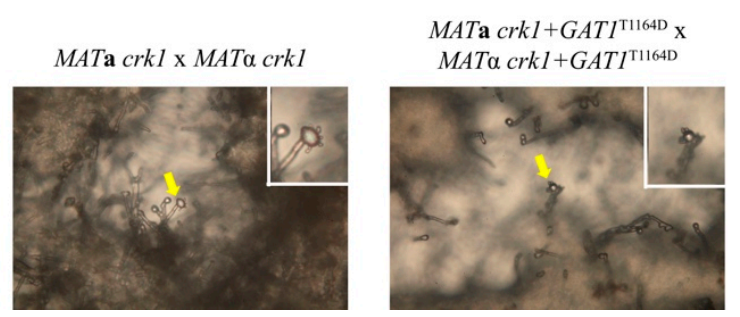

Figure 9. The GAT1 ${ }^{\mathrm{T} 1164 \mathrm{D}}$ phospho-mimetic active allele dramatically repressed dikaryotic filamentation in the wild-type cross but showed slight effects on mating under crk1 mutant background. (A) C. neoformans MATa and MAT $\alpha$ strains were crossed as indicated. Mating was conducted on V8 agar plates and incubated at $26{ }^{\circ} \mathrm{C}$ in the dark. Photos were taken $16 \mathrm{~h}$ and $24 \mathrm{~h}$ post-incubation at $100 \times$ magnification. (B) Small photos demonstrate the basidia in the $c r k 1$ and $c r k 1 G A T 1^{\mathrm{T} 1164 \mathrm{D}}$ bilateral mutant crosses respectively. Yellow arrow indicates basidium. Photos were taken $24 \mathrm{~h}$ post-incubation at $400 \times$ magnification. 
A

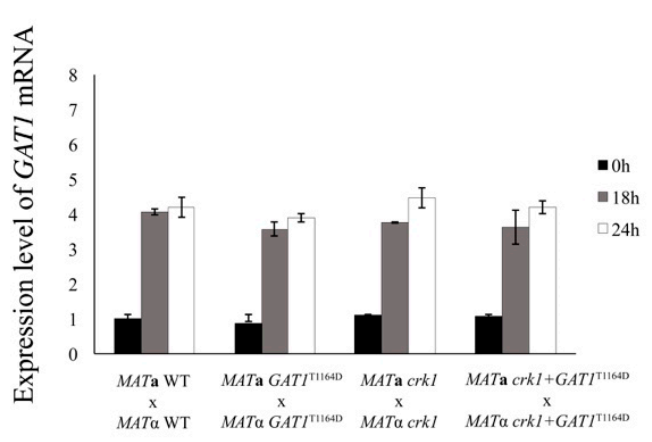

$\mathrm{C}$

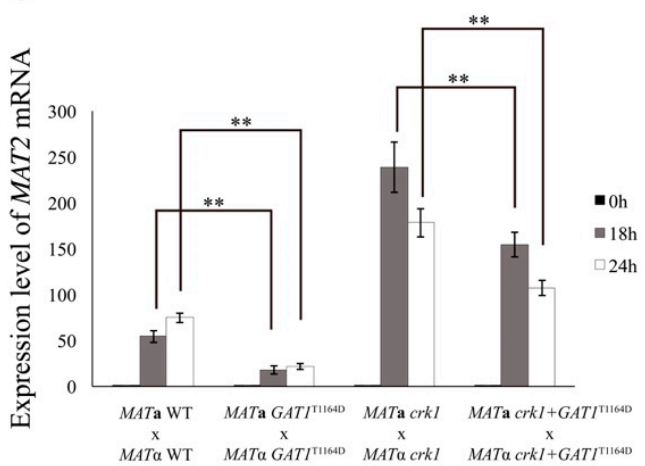

$\mathrm{E}$

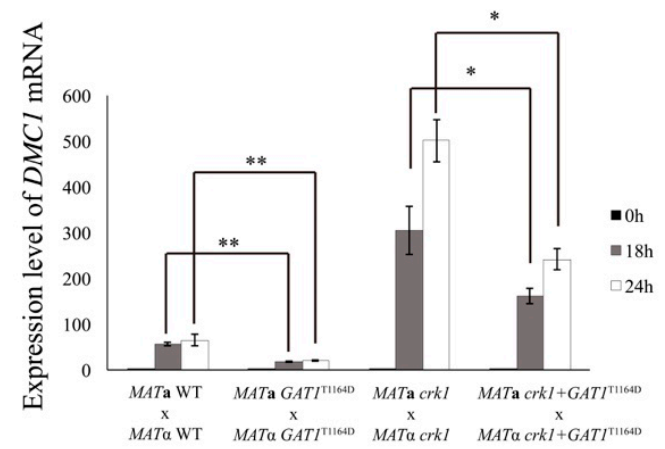

B

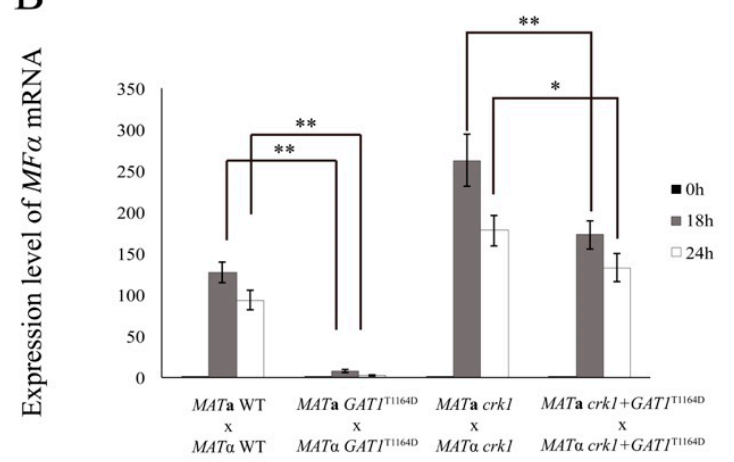

$\mathrm{D}$

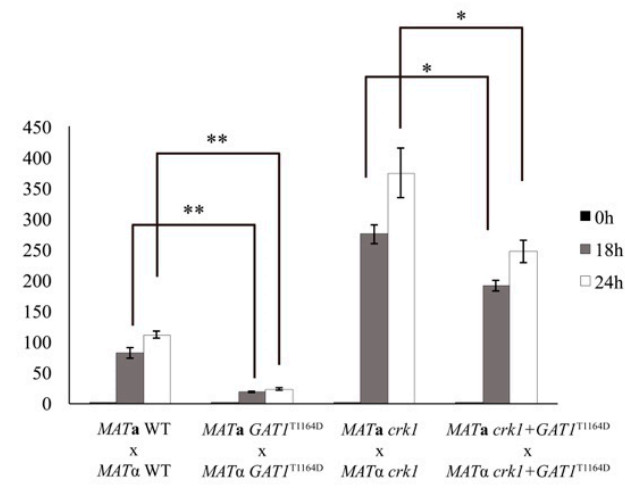

Figure 10. The expression of mating-related genes was down-regulated by the GAT1 phospho-mimetic allele. C. neoformans MATa and MAT $\alpha$ strains were crossed as indicated. Mating was conducted on V8 agar plates and incubated at $26^{\circ} \mathrm{C}$ in the dark. Samples were collected at 0,18 , and $24 \mathrm{~h}$ post-incubation. The expression of GAT1 (A), MF $\alpha$ (B), MAT2 (C), CSA1 (D), and DMC1 (E) was examined by real-time qRT-PCR analysis. Triplicate reactions for each sample were conducted. Error bar represents the standard deviation from the mean of three replicates. The results were normalized to C. neoformans GPD1 expression. (** indicates $p<0.005 ; *$ indicates $p<0.05)$.

\subsection{Deletion of GAT1 Partially Restored Dikaryotic Filamentation of CRK1 Overexpression Strain}

Previous study showed that CRK1 overexpression represses the formation of dikaryotic filament and also reduces the expression levels of the mating-related genes [29]. In this study, we revealed that GAT1 may be one of the downstream targets of CRK1. We hypothesized that deletion of GAT1 under the CRK1 overexpression background may restore the production of dikaryotic filament. To address this question, we deleted the GAT1 gene in the MATa and MAT $\alpha$ CRK1 overexpression strains and bisexual mating was then conducted on V8 medium. After $24 \mathrm{~h}$ incubation, dikaryotic filamentation was repressed dramatically in the bilateral cross of CRK1 overexpression strains (Figure S7). As expected, filamentation was partially restored in the bilateral cross involved the MATa and MAT $\alpha$ CRK1 
overexpression + gat1 mutants. However, the level of filamentation was much less than those observed in the wild-type and bilateral gat1 mutant cross (Supplementary Figure S7).

Gene expression analysis was further performed to confirm the phenotypic observation. Mating samples were harvested at 18 and $24 \mathrm{~h}$ and the transcript levels of $M F \alpha$ and $M A T 2$ were examined. At 18 and $24 \mathrm{~h}$, the $M F \alpha$ expression levels, $56 \pm 12.93$ and $39.8 \pm 8.57$ fold respectively, in the bilateral CRK1 overexpression + gat1 mutant cross were higher than those in the bilateral CRK1 overexpression cross (Figure S8A). The MAT2 mRNA levels, $21.6 \pm 0.97$ fold at $18 \mathrm{~h}$ and $28.9 \pm 5.59$ fold at $24 \mathrm{~h}$, in the bilateral $C R K 1$ overexpression + gat1 mutant cross were also higher than those in the bilateral CRK1 overexpression cross (Figure S8B). However, the expression levels of these two mating-related genes in the bilateral CRK1 overexpression + gat1 mutant cross were not as high as the levels seen in the wild-type and bilateral gat1 mutant crosses (Supplementary Figure S8A,B). Taken together, our results demonstrated that deletion of GAT1 can only partially restore the repression of dikaryotic filamentation and gene expression conferred by CRK1 overexpression, suggesting that Gat1 may not be the only downstream target of Crk1 to regulate bisexual mating.

\subsection{C. neoformans CRK1 Coordinated with GAT1 to Repress Bisexual Differentiation}

According to our previous results, deletion of C. neoformans GAT1 enhanced the formation of dikaryotic filaments and increased the mRNA level of $M F \alpha$ pheromone gene. To examine the effect of GAT1 overexpression on bisexual differentiation, we expressed GAT1 under the C. neoformans GPD1 constitutive promoter in the JEC20 and JEC21 strains. The GAT1 transcript level was markedly increased in the bisexual cross of GAT1 overexpression strains (Supplementary Figure S5A). However, no discernible phenotype was observed when compared to the wild-type cross (Supplementary Figure S4), and $M F \alpha$ expression level was similar to that in the wild-type cross (Supplementary Figure S5B). Hence, the overexpression of GAT1 possibly had no effect on bisexual mating.

Previous studies demonstrated that the degree of bisexual mating inhibition depends on CRK1 expression [30]. To examine whether overexpression of GAT1 required the proper level of CRK1 to exhibit the filament inhibition phenotype, we tried to elevate the expression levels of CRK1 and GAT1 under the GPD1 promoter in the MAT $\alpha$ crk1 strain. We transformed $P_{G P D 1}:$ CRK1 plasmid into the $M A T \alpha$ crk1 mutant and selected the transformants which showed filamentation phenotypes like the wild-type strains when crossed with the MATa wild-type or crk1 mutant strain. We then further transformed $\mathrm{P}_{G P D 1}:: G A T 1$ construct into the $M A T \alpha$ crk1 $+\mathrm{P}_{G P D 1}:: C R K 1$ strain to generate the $M A T \alpha$ $c r k 1+\mathrm{P}_{G P D 1}:: C R K 1+\mathrm{P}_{G P D 1}:: G A T 1$ strain. Bisexual mating was conducted on V8 agar medium for $24 \mathrm{~h}$. Dikaryotic filamentation of the $M A T \alpha c r k 1+\mathrm{P}_{G P D 1}:: C R K 1$ was similar to that in the wild-type and $M A T \alpha$ crk1 unilateral mutant cross (Supplementary Figure S6A-C), and the mRNA levels of GAT1 and $M F \alpha$ in the $M A T \alpha c r k 1+\mathrm{P}_{G P D 1}:: C R K 1$ unilateral cross were similar and those in the wild-type cross (Supplementary Figure S9A,B). Interestingly, dikaryotic filamentation was greatly decreased in the $M A T \alpha c r k 1+\mathrm{P}_{G P D 1}:: C R K 1+\mathrm{P}_{G P D 1}:: G A T 1$ strain (Supplementary Figure S6D), and the expression level of $M F \alpha$ was significantly decreased in the $M A T \alpha$ crk1 $+\mathrm{P}_{G P D 1}:: C R K 1+\mathrm{P}_{G P D 1}:$ GAT1 cross (Supplementary Figure S9B). Thus, C. neoformans CRK1 likely coordinated with GAT1 to negatively regulate the process of bisexual mating.

\section{Discussion}

The evolutionarily flexible functions of fungal Ime2/Crk1 kinases reported in several fungal species play an important role in sexual differentiation and also regulate programmed cell death, endosome motility, extracellular protease activity, mycotoxin production, and cellulase production [42-44,60-62]. Previous studies indicated that $C$. neoformans CRK1 plays a negative role in cell fusion and pheromone expression in bisexual mating [29]. In this study, we provided a detailed observation for dikaryotic filamentation and demonstrated that the formation of basidia and basidiospores in the bilateral crk1 mutant cross was earlier than the wild-type cross by approximately $18 \mathrm{~h}$. The length of dikaryotic filaments in the bilateral crk1 mutant cross was shorter but still maintained typical fused clamp cells 
and normal nuclear distribution. Taken together, our results revealed that C. neoformans CRK1 was involved in maintaining proper dikaryotic filamentation and modulating the occurrence of karyogamy and meiosis to regulate accurate chronology in bisexual mating process. By bioinformatic search for the C. neoformans genes containing Ime 2 consensus phosphorylation RPGT $^{1164}$ site, we identified the GAT1 gene as a putative target of $C$. neoformans Crk1. Our functional studies revealed that GAT1 played a negative role in the transcription of MAT2 and pheromone production, and the crk1gat1 double mutant strain phenotypically copied the mating phenotypes of the crk1 mutant in bisexual differentiation. Mutations of RPGT ${ }^{1164}$ in Gat1 resulted in dramatic phenotypes of bisexual mating, indicating that $\mathrm{RPGT}^{1164}$ sequence played an important function of Gat1 and was essential for proper regulation of bisexual mating. We demonstrated that both $C$. neoformans CRK1 and GAT1 formed a regulatory circuit to negatively modulate MAT2 and other potential unidentified targets for pheromone production and other processes in bisexual mating.

It is interesting to observe shorter dikaryotic filaments and earlier basidium formation associated with the crosses involved crk1 mutants. Early basidium formation was also reported in the C. neoformans mutant deleting the microtubule binding BIM1 gene; however, mutation of BIM1 also results in loss of filament integrity [63]. In contrast to the bim1 mutant, the features of dikaryotic filaments in the bilateral crk1 mutant cross, such as straight filament and typical clamp cell, did not differ from those in the wild-type cross (Figure 1). Moreover, the localization and distribution of nuclei in the bilateral crk1 mutant cross were the same as the wild-type cross. These findings suggest that the involvement of C. neoformans CRK1 gene in sexual development might be different from the BIM1 gene.

Mitotic progression is one of the mechanisms affecting cell size or filamentation in fungi $[64,65]$. Studies of the cell cycle machinery in S. pombe provide some insights for our study [66]. The S. pombe wee1 gene is a mitotic inhibitor that controls G2/M transition. Deletion of the wee1 gene decreases the period in G2 phase and causes early entry into mitosis, thus the cell size of wee1 mutant is smaller than the wild-type [67]. Recently, the S. pombe wee1 homologues were identified in other fungi, such as $U$. maydis wee1 and Beauveria bassiana wee1. Mutation of wee1 results in short filaments in U. maydis and B. bassiana [68,69]. Furthermore, S. pombe cdc13 gene is an important B-type cyclin that promotes G2 phase to enter mitosis during cell cycle [70,71]. In $U$. maydis, overexpression of $c l b 2$, the S. pombe cdc13 homologue, also results in shorter G2 phase and smaller cell size [72]. We assume that C. neoformans Crk1 may also regulate cell cycle transition. Preliminary bioinformatic survey in C. neoformans genome revealed the wee1 homologues, CNG02130 and CNG03960, and several putative kinases which are related to cell cycle progression may be potential substrates of Crk1. The phenotypes exhibited by the $c r k 1$ mutants may be caused by unscheduled cell-cycle progression. Whether Crk1 is involved in cell cycle progression in $C$. neoformans and how it connects to the sexual processes such as dikaryotic filamentation and basidium formation require further investigation.

The expression of CRK1 is lower during early stage of bisexual mating process [29]. We confirmed similar results in this study and also found the CRK1 mRNA level was slightly elevated between 16 to $18 \mathrm{~h}$ post-incubation (Figure 3). Thus, CRK1 may play a significant role during later stage of mating and dikaryotic filamentation stage. To further reveal the role of CRK1 in the bisexual mating process, we determined the expression of genes related to mating, filamentation, karyogamy, and meiosis in the bilateral crk1 mutant cross. The expression of these genes was increased in the bilateral crk1 mutant cross, which is consistent with previous conclusion that CRK1 has a negative role in bisexual mating. Furthermore, as the CRK1 mRNA level increased around $18 \mathrm{~h}$ in the wild-type cross, the expression of all the genes tested was also increased greatly at this stage in the bilateral crk1 mutant cross (Figure 3). Elevated mRNA levels of KAR7 and DMC1 supported the phenotypic observation of early initiation of karyogamy and meiosis in the bilateral crk1 mutant cross (Figures 1 and 3E,F) These findings indicated that the $C$. neoformans $C R K 1$ gene might regulate multiple signal transduction events in bisexual mating.

MAT2 and ZNF2 are two major transcriptional regulators that control cell fusion and filamentation in C. neoformans. We further addressed the genetic relationship between CRK1, MAT2 and ZNF2. It was hypothesized that the deletion of the negative regulator $C R K 1$ could bypass the non-filamentation 
phenotype in the bilateral mat 2 or znf2 mutant cross. Interestingly, dikaryotic filamentation was blocked in the bilateral znf2crk1 double mutant cross (Supplementary Figure S2A). However, the expression level of CRK1 was not altered in the bilateral znf2 mutant cross (Supplementary Figure S2C). These findings suggested that $C$. neoformans ZNF2 possibly functioned downstream of CRK1 in bilateral mating, thus resulting in non-filamentation phenotype in the bilateral crk1znf2 mutant cross. In addition, dikaryotic filamentation was also blocked in the bilateral mat2crk1 mutant cross; however, the expression of CRK1 was decreased significantly in the bilateral mat 2 mutant cross and increased in the MAT2-overexpressed strain cross (Figures 4 and 5D). In S. cerevisiae, IME1 activates the expression of IME2 at the initiation stage of meiosis, and then phosphorylation of Ime1 by Ime2 leads to the degradation of Ime1 for the completion of meiotic process [73-75]. Previous study showed that C. neoformans Mat2 regulates pheromone responsive genes by binding to the pheromone response element (PRE; AAAGAACAAAAAGACA) in their promoter [5]. An example is the C. neoformans GPA3 gene which contains one PRE element at its promoter sequence and acts as a negative regulator of sexual differentiation [5]. We searched $1000 \mathrm{bp}$ region upstream the CRK1 open reading frame for the enriched motifs by the MEME algorithm, and one putative PRE (GGAAAAGAAAAGGTAT) was identified in the CRK1 promoter. Along with the analysis of CRK1 promoter sequence and our gene expression data, these findings suggest that MAT2 might regulate $C R K 1$ expression during bisexual mating process.

C. neoformans Crk1 is a Ser/Thr protein kinase containing TXY activation motif. In this study, we predicted the potential targets of $C$. neoformans $C r k 1$ based on Ime2 consensus phosphorylation site and the GATA transcription factor Gat1 was identified and characterized. The bilateral mutant crosses involved the gat1 deletion mutants showed increased aerial hyphal formation and elevated expression of mating-related genes (Figures 6A and 7). Strains containing the phospho-null GAT1 allele in the predicted Ime2 consensus phosphorylation site also showed similar phenotypes as the gat1 deletion mutants (Figure 6B). Our genetic and phenotypic findings indicated that C. neoformans GAT1 gene modulated the sexual processes such as regulation of pheromone expression and aerial hyphae formation, which are overlapped with the roles of CRK1 during bisexual mating. Interestingly, GAT1 and CRK1 played divergent role in basidia formation, as the deletion of C. neoformans GAT1 gene did not result in early appearance of basidia during bisexual mating. In addition, C. neoformans wild-type and crk1 mutant strains containing the GAT1 phospho-mimetic allele both exhibited reduction of dikaryotic filamentation (Figure 9) and gene expression results also supported the roles of GAT1 during bisexual mating (Figure 10). Thus, our data supported that $C$. neoformans Gat1, as one of the phosphorylation substrates of Crk1, also played a negative role in bisexual mating.

In our expression data, the transcript levels of the HMG transcription factor MAT2 and MF $\alpha$ pheromone genes were both elevated in the bilateral crk1 and gat 1 mutant crosses (Figures 3 and 7). Moreover, CRK1 was downregulated in the bilateral mat2 mutant cross (Figure 4C). Studies in S. pombe indicated that the HMG family transcription factor ste11 is a key regulator during sexual differentiation $[56,76]$. Under nitrogen starvation, the S. pombe GATA type transcription factor Gaf1, the homologue of $C$. neoformans GAT1 gene, downregulates the expression of ste11 via binding to the ste11 promoter region. Deletion of gaf1 results in increased ste11 mRNA level [55]. Fungal GATA type transcription factors have also been demonstrated to recognize and bind to the consensus sequence, $5^{\prime}$-(T/A/C) GATA(A/G)-3', of the promoter regions under regulation [76,77]. We initially analyzed and identified one putative binding site ( $5^{\prime}$-CGATAA- $\left.3^{\prime}\right)$ in the $1 \mathrm{~Kb}$ upstream region of the MAT2 promoter sequence. Thus, we hypothesize that CRK1 was transcriptionally activated by MAT2, then Crk1 phosphorylated and activated Gat1, and then Gat1 repressed the expression of MAT2 during bisexual mating process in C. neoformans. However, further studies for the evidences of the transcriptional and translational regulation are needed to support this regulatory circuit.

Based on our studies, we propose a potential regulatory circuit to illustrate how Crk1 and Gat1 regulate bisexual mating via the key transcriptional regulator Mat2 in C. neoformans (Figure 11). C. neoformans CRK1 gene regulates pheromone production, formation and elongation of dikaryotic 
filaments and basidium formation. During the bisexual mating process, a pheromone is first sensed and transduced through the Cpk1-MAPK signaling pathway to activate the downstream transcription factor MAT2. MAT2 plays a key role to modulate various sexual events including promoting cell fusion process and regulating ZNF2 expression to induce dikaryotic filamentation. Based on our studies, we propose a regulatory circuit to describe how Crk1-Gat1 modulate MAT2 activity for mating regulation. Upon pheromone induction, MAT2 is activated via conserved signaling events to induce the expression of various genes including CRK1. Crk1 kinase regulates Gat1 activity by post-translational phosphorylation. The activated Gat1 will in turn inhibit MAT2 expression to modulate bisexual mating processes (Figure 11). Since GAT1 deletion and alleles with modified phosphorylation site could only partially suppress the filamentation phenotypes of $c r k 1$ or wild-type cross (Figure 9), we hypothesize that GAT1 possibly is not the only downstream target of Crk1 for mating regulation. Future study is needed to further elucidate the complicated regulatory networks mediated by Crk1.

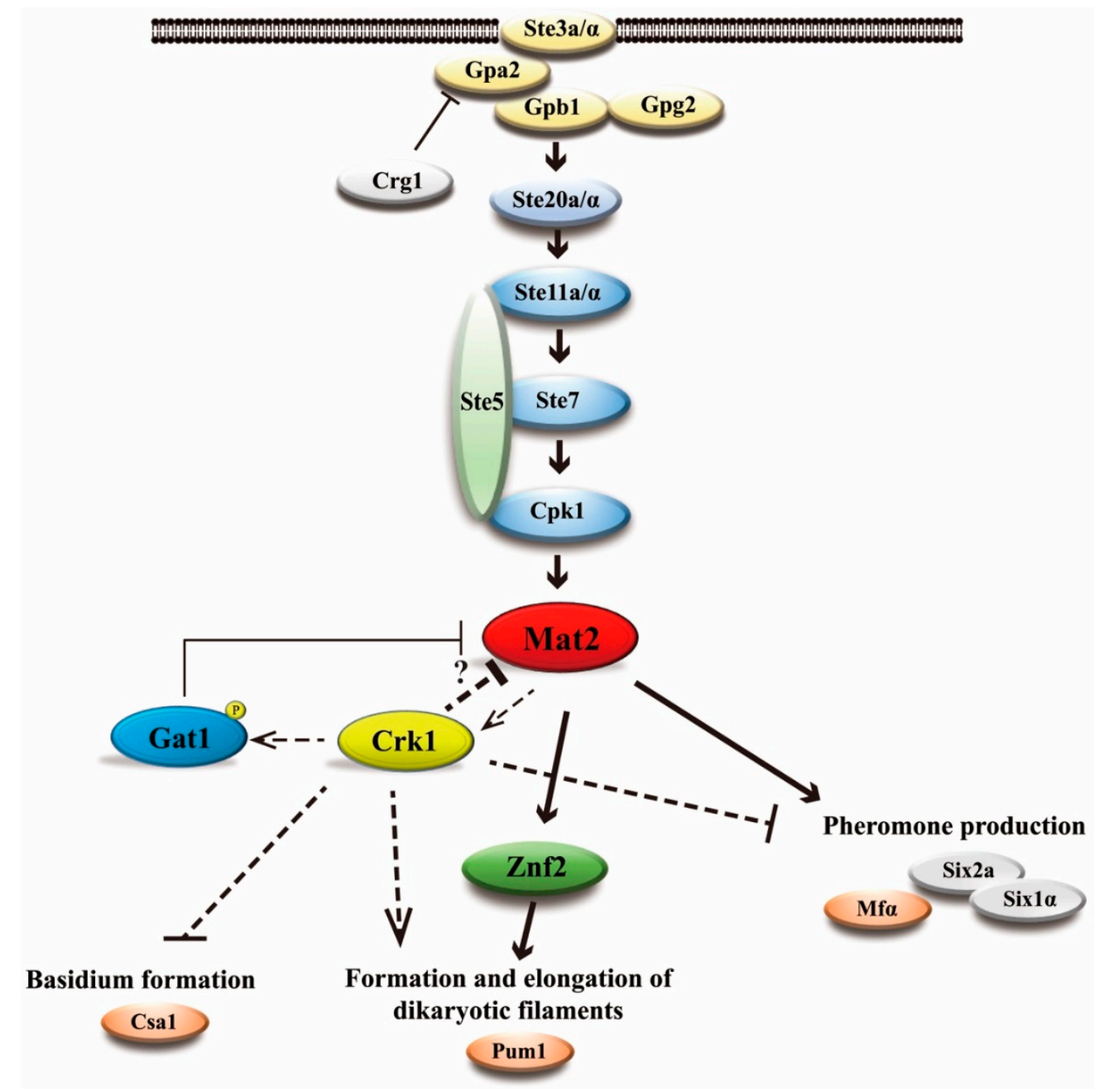

Figure 11. C. neoformans Crk1 and Gat1 negatively regulated the expression of Mat2 to coordinately modulate sexual differentiation. Mat2 is a major transcription factor downstream the Cpk1-MAPK signaling pathway to regulate pheromone production and mating responses in C. neoformans. The protein kinase Crk1 negatively regulated MAT2 expression via phosphorylating Gat1 or other target(s) to reduce the MAT2 transcript level and repress pheromone production and other mating responses. The expression of CRK1 was possibly also regulated by Mat2. Crk1-Gat1 may form a regulatory circuit to properly regulate MAT2/Mat2 levels to control the progression of sexual filamentation and transition to basidium stage. We propose this model how Crk1 regulates pheromone production, elongation of dikaryotic filaments, and basidium formation during bisexual mating. 
Supplementary Materials: The following are available online at http://www.mdpi.com/2073-4425/11/6/669/s1; Figure S1. Dikaryotic filamentation was examined in the wild-type and bilateral $c r k 1$ mutant crosses. Co-incubation of (A) MATa wild-type $\mathrm{P}_{G P D 1}:: G F P-H 2 B$ and MAT $\alpha$ wild-type strains and (B) MATa $c r k 1 \mathrm{P}_{G P D 1}:: G F P-H 2 B$ and $M A T \alpha c r k 1$ mutants was conducted on V8 agar plates incubated at $26^{\circ}$ in the dark. Colony edges of mating mixtures were photographed from 16 to $72 \mathrm{~h}$ post-incubation at $100 \times$ magnification. Figure S2. ZNF2 mutation did not affect CRK1 expression, but blocked dikaryotic filamentation in the bilateral crk1 mutant cross. (A) $C$. neoformans $M A T \mathbf{a}$ and MAT $\alpha$ strains were crossed as indicated. Mating was conducted on V8 agar plates incubated at $26^{\circ} \mathrm{C}$ in the dark. Photos were taken $24 \mathrm{~h}$ post-incubation at $100 \times$ magnification. Bilateral crosses of the MATa and MAT $\alpha$ wild-type and znf2 mutants were conducted. Samples were collected at 0, 6, 18 and $24 \mathrm{~h}$ post-incubation and subjected to gene expression analysis. $M F \alpha(\mathbf{B})$ and CRK1 (C) expression during bisexual mating was examined by real-time qRT-PCR analysis. Triplicate reactions for each sample were conducted. Error bar represents the standard deviation from the mean of three replicates. The results were normalized to C. neoformans GPD1 expression. (* indicates $p<0.005)$. Figure S3. The expression level of mating-related genes were upregulated slightly in the bilateral crk1gat1 mating cross. Bilateral crosses involved the MATa and MAT $\alpha$ wild-type, gat1, crk1, and crk1gat1 mutants were conducted on V8 agar plates and incubated at $26^{\circ} \mathrm{C}$ in the dark. Samples were collected at 0,18 , and $24 \mathrm{~h}$ post-incubation. The expression of $M F \alpha(\mathbf{A}), M A T 2$ (B), CSA1 (C), and DMC1 (D) was examined by real-time qRT-PCR analysis. Triplicate reactions for each sample were conducted. Error bar represents the standard deviation from the mean of three replicates. The results were normalized to C. neoformans GPD1 expression. (* indicates $p<0.05$ ). Figure S4. Overexpression of GAT1 did not repress dikaryotic filamenation. C. neoformans $M A T \mathbf{a}$ and $M A T \alpha$ strains GAT1 overexpression strains were crossed as indicated. Mating was conducted on V8 agar plates at $26{ }^{\circ} \mathrm{C}$ in the dark. Photos were taken at $24 \mathrm{~h}$ post-incubation at $100 \times$ magnification. Figure S5. The transcription level of $M F \alpha$ in the GAT1 overexpression strains cross was similar to that in the wild-type cross. C. neoformans MATa and MAT $\alpha$ wild-type and GAT1 overexpression strains were crossed and incubated on V8 agar plates at $26^{\circ} \mathrm{C}$ in the dark. Samples were collected at 0 and $24 \mathrm{~h}$ post-incubation. The expression of GAT1 (A) and $M F \alpha(\mathbf{B})$ was examined by real-time qRT-PCR analysis. Triplicate reactions for each sample were conducted. Error bar represents the standard deviation from the mean of three replicates. The results were normalized to C. neoformans GPD1 expression. Figure S6. Dikaryotic filamentation was reduced with overexpression of GAT1 and CRK1. C. neoformans MATa and MAT $\alpha$ strains were crossed as indicated. Mating was conducted on V8 agar plates at $26^{\circ} \mathrm{C}$ in the dark. Photos were taken at $24 \mathrm{~h}$ post-incubation at $100 \times$ magnification. Figure S7. Deletion of GAT1 partially recovered sexual filamentation of bilateral CRK1 overexpression cross. C. neoformans MATa and $M A T \alpha$ strains were crossed as indicated. Mating was conducted on V8 agar plates at $26{ }^{\circ} \mathrm{C}$ in the dark. Photos were taken at $24 \mathrm{~h}$ post-incubation at $100 \times$ magnification. Figure S8. GAT1 negatively regulated MF $\alpha$ and $M A T 2$ gene expression during mating process. Bilateral crosses involved the MATa and MAT $\alpha$ wild-type, gat1 mutants, $\mathrm{P}_{G P D 1}:: C R K 1$ overexpression strains, and $\mathrm{P}_{G P D 1}:: C R K 1+$ gat1 mutant strains were conducted on V8 agar plates at $26^{\circ} \mathrm{C}$ in the dark. Samples were collected at 0,18 , and 24 h post-incubation. The expression of $M F \alpha(\mathbf{A})$ and MAT2 (B) was examined by real-time qRT-PCR analysis. Error bar represents the standard deviation from the mean of three replicates.Triplicate reactions for each sample were conducted. The results were normalized to C. neoformans GPD1 expression. ( ${ }^{*}$ indicates $p<0.05 ;{ }^{*}$ indicates $\left.p<0.005\right)$. Figure S9. Overexpression of GAT1 and $C R K 1$ reduced $M F \alpha$ expression during bisexual mating. Bilateral crosses involved the MATa and MAT $\alpha$ wild-type, crk1, gat1, and GAT1 ${ }^{\mathrm{T} 1164 \mathrm{~A}}$ mutants, and MATa wild-type crossed with MAT $\alpha$ crk1 + $\mathrm{P}_{G P D 1}:: C R K 1$, and MAT $\alpha$ $c r k 1+\mathrm{P}_{G P D 1}:: C R K 1+\mathrm{P}_{G P D 1}:: G A T 1$ strains were conducted on V8 agar plates at $26{ }^{\circ} \mathrm{C}$ in the dark. Samples were collected at 0 and $24 \mathrm{~h}$ post-incubation and the expression of GAT1 (A) and MF $\alpha$ (B) was examined by real-time qRT-PCR analysis. Triplicate reactions for each sample were conducted. Error bar represents the standard deviation from the mean of three replicates. The results were normalized to C. neoformans GPD1 expression. Table S1. Oligonucleotides used in this study. Table S2. Potential transcription factor targets of $C$. neoformans Crk1.

Author Contributions: Conceptualization, K.-H.L. and W.-C.S.; Data curation, K.-H.L. and W.-C.S.; Formal analysis, K.-H.L.; Funding acquisition, W.-C.S.; Investigation, K.-H.L.; Methodology, K.-H.L.; Validation, K.-H.L.; Writing-original draft, K.-H.L. and W.-C.S.; Writing-review \& editing, K.-H.L. and W.-C.S. All authors have read and agreed to the published version of the manuscript.

Funding: We thank the GenoInfo Core Facility (C1) funded by NCFPB of MOST Taiwan (MOST106-2319-B-010-001) for bioinformatics analysis. This work was supported by National Science Council Grant MOST103-2311-B-002-024.

Acknowledgments: We thank the genome sequence information provided at Stanford Genome Technology Center, the Institute for Genome Research, the Broad Institute, and Duke University. We are grateful to Technology Commons, College of Life Science, NTU (Taiwan) for Delta Vision Core.

Conflicts of Interest: The authors declare no conflicts of interest.

\section{References}

1. Hull, C.M.; Heitman, J. Genetics of Cryptococcus neoformans. Annu. Rev. Genet. 2002, 36, 557-615. [CrossRef] [PubMed]

2. Idnurm, A.; Bahn, Y.S.; Nielsen, K.; Lin, X.; Fraser, J.A.; Heitman, J. Deciphering the model pathogenic fungus Cryptococcus neoformans. Nat. Rev. Microbiol. 2005, 3, 753-764. [CrossRef] [PubMed] 
3. Kwon-Chung, K.J.; Bennett, J.E. High prevalence of Cryptococcus neoformans var. gattii in tropical and subtropical regions. Zentralbl. Bakteriol. Mikrobiol. Hyg. A 1984, 257, 213-218. [CrossRef]

4. Kozubowski, L.; Heitman, J. Profiling a killer, the development of Cryptococcus neoformans. FEMS Microbiol. Rev. 2012, 36, 78-94. [CrossRef] [PubMed]

5. Kruzel, E.K.; Giles, S.S.; Hull, C.M. Analysis of Cryptococcus neoformans sexual development reveals rewiring of the pheromone-response network by a change in transcription factor identity. Genetics 2012, 191, 435-449. [CrossRef] [PubMed]

6. Perfect, J.R.; Wong, B.; Chang, Y.C.; Kwon-Chung, K.J.; Williamson, P.R. Cryptococcus neoformans: Virulence and host defences. Med. Mycol. 1998, 36 (Suppl. 1), 79-86. [PubMed]

7. Zhao, Y.; Lin, J.; Fan, Y.; Lin, X. Life cycle of Cryptococcus neoformans. Annu. Rev. Microbiol. 2019, 73, 17-42. [CrossRef]

8. Hsueh, Y.P.; Shen, W.C. A homolog of Ste6, the a-factor transporter in Saccharomyces cerevisiae, is required for mating but not for monokaryotic fruiting in Cryptococcus neoformans. Eukaryot. Cell 2005, 4, 147-155. [CrossRef]

9. Hsueh, Y.P.; Xue, C.; Heitman, J. G protein signaling governing cell fate decisions involves opposing G $\alpha$ subunits in Cryptococcus neoformans. Mol. Biol. Cell 2007, 18, 3237-3249. [CrossRef] [PubMed]

10. Nichols, C.B.; Fraser, J.A.; Heitman, J. PAK kinases Ste20 and Pak1 govern cell polarity at different stages of mating in Cryptococcus neoformans. Mol. Biol. Cell 2004, 15, 4476-4489. [CrossRef]

11. Shen, W.C.; Davidson, R.C.; Cox, G.M.; Heitman, J. Pheromones stimulate mating and differentiation via paracrine and autocrine signaling in Cryptococcus neoformans. Eukaryot. Cell 2002, 1, 366-377. [CrossRef] [PubMed]

12. Wang, P.; Perfect, J.R.; Heitman, J. The G-protein beta subunit GPB1 is required for mating and haploid fruiting in Cryptococcus neoformans. Mol. Cell. Biol. 2000, 20, 352-362. [CrossRef] [PubMed]

13. Clarke, D.L.; Woodlee, G.L.; McClelland, C.M.; Seymour, T.S.; Wickes, B.L. The Cryptococcus neoformans STE11 $\alpha$ gene is similar to other fungal mitogen-activated protein kinase kinase kinase (MAPKKK) genes but is mating type specific. Mol. Microbiol. 2001, 40, 200-213. [CrossRef] [PubMed]

14. Davidson, R.C.; Nichols, C.B.; Cox, G.M.; Perfect, J.R.; Heitman, J. A MAP kinase cascade composed of cell type specific and non-specific elements controls mating and differentiation of the fungal pathogen Cryptococcus neoformans. Mol. Microbiol. 2003, 49, 469-485. [CrossRef] [PubMed]

15. Feretzaki, M.; Heitman, J. Genetic circuits that govern bisexual and unisexual reproduction in Cryptococcus neoformans. PLoS Genet. 2013, 9, e1003688. [CrossRef]

16. Lin, X.; Jackson, J.C.; Feretzaki, M.; Xue, C.; Heitman, J. Transcription factors Mat2 and Znf2 operate cellular circuits orchestrating opposite- and same-sex mating in Cryptococcus neoformans. PLoS Genet. 2010, 6, e1000953. [CrossRef]

17. Hull, C.M.; Boily, M.J.; Heitman, J. Sex-specific homeodomain proteins Sxi1 $\alpha$ and Sxi2a coordinately regulate sexual development in Cryptococcus neoformans. Eukaryot. Cell 2005, 4, 526-535. [CrossRef]

18. Ekena, J.L.; Stanton, B.C.; Schiebe-Owens, J.A.; Hull, C.M. Sexual development in Cryptococcus neoformans requires CLP1, a target of the homeodomain transcription factors Sxi1 $\alpha$ and Sxi2a. Eukaryot. Cell 2008, 7, 49-57. [CrossRef]

19. Wang, L.; Zhai, B.; Lin, X. The link between morphotype transition and virulence in Cryptococcus neoformans. PLoS Pathog. 2012, 8, e1002765. [CrossRef]

20. Kaur, J.N.; Panepinto, J.C. Morphotype-specific effector functions of Cryptococcus neoformans PUM1. Sci. Rep. 2016, 6, 23638. [CrossRef]

21. Wang, L.; Tian, X.; Gyawali, R.; Upadhyay, S.; Foyle, D.; Wang, G.; Cai, J.J.; Lin, X. Morphotype transition and sexual reproduction are genetically associated in a ubiquitous environmental pathogen. PLoS Pathog. 2014, 10, e1004185. [CrossRef] [PubMed]

22. Lin, J.; Zhao, Y.; Ferraro, A.R.; Yang, E.; Lewis, Z.A.; Lin, X. Transcription factor Znf2 coordinates with the chromatin remodeling SWI/SNF complex to regulate cryptococcal cellular differentiation. Commun. Biol. 2019, 2, 412. [CrossRef] [PubMed]

23. Lee, S.C.; Heitman, J. Function of Cryptococcus neoformans KAR7 (SEC66) in karyogamy during unisexual and opposite-sex mating. Eukaryot. Cell 2012, 11, 783-794. [CrossRef] [PubMed] 
24. Fu, C.; Heitman, J. PRM1 and KAR5 function in cell-cell fusion and karyogamy to drive distinct bisexual and unisexual cycles in the Cryptococcus pathogenic species complex. PLoS Genet. 2017, 13, e1007113. [CrossRef] [PubMed]

25. Liu, L.; He, G.J.; Chen, L.; Zheng, J.; Chen, Y.; Shen, L.; Tian, X.; Li, E.; Yang, E.; Liao, G.; et al. Genetic basis for coordination of meiosis and sexual structure maturation in Cryptococcus neoformans. eLife 2018, 7, e38683. [CrossRef] [PubMed]

26. Idnurm, A.; Heitman, J. Light controls growth and development via a conserved pathway in the fungal kingdom. PLoS Biol. 2005, 3, e95. [CrossRef] [PubMed]

27. Lu, Y.K.; Sun, K.H.; Shen, W.C. Blue light negatively regulates the sexual filamentation via the Cwc1 and Cwc2 proteins in Cryptococcus neoformans. Mol. Microbiol. 2005, 56, 480-491. [CrossRef]

28. Yeh, Y.L.; Lin, Y.S.; Su, B.J.; Shen, W.C. A screening for suppressor mutants reveals components involved in the blue light-inhibited sexual filamentation in Cryptococcus neoformans. Fungal Genet. Biol. 2009, 46, 42-54. [CrossRef]

29. Liu, K.H.; Shen, W.C. Mating differentiation in Cryptococcus neoformans is negatively regulated by the Crk1 protein kinase. Fungal Genet. Biol. 2011, 48, 225-240. [CrossRef]

30. Smith, H.E.; Mitchell, A.P. A transcriptional cascade governs entry into meiosis in Saccharomyces cerevisiae. Mol. Cell. Biol. 1989, 9, 2142-2152. [CrossRef]

31. Honigberg, S.M.; Purnapatre, K. Signal pathway integration in the switch from the mitotic cell cycle to meiosis in yeast. J. Cell Sci. 2003, 116, 2137-2147. [CrossRef] [PubMed]

32. Kassir, Y.; Adir, N.; Boger-Nadjar, E.; Raviv, N.G.; Rubin-Bejerano, I.; Sagee, S.; Shenhar, G. Transcriptional regulation of meiosis in budding yeast. Int. Rev. Cytol. 2003, 224, 111-171. [PubMed]

33. Clifford, D.M.; Marinco, S.M.; Brush, G.S. The meiosis-specific protein kinase Ime2 directs phosphorylation of replication protein A. J. Biol. Chem. 2004, 279, 6163-6170. [CrossRef] [PubMed]

34. Clifford, D.M.; Stark, K.E.; Gardner, K.E.; Hoffmann-Benning, S.; Brush, G.S. Mechanistic insight into the Cdc28-related protein kinase Ime2 through analysis of replication protein A phosphorylation. Cell Cycle 2005, 4, 1826-1833. [CrossRef] [PubMed]

35. Holt, L.J.; Hutti, J.E.; Cantley, L.C.; Morgan, D.O. Evolution of Ime2 phosphorylation sites on Cdk1 substrates provides a mechanism to limit the effects of the phosphatase Cdc14 in meiosis. Mol. Cell 2007, 25, 689-702. [CrossRef] [PubMed]

36. Howard, C.J.; Hanson-Smith, V.; Kennedy, K.J.; Miller, C.J.; Lou, H.J.; Johnson, A.D.; Turk, B.E.; Holt, L.J. Ancestral resurrection reveals evolutionary mechanisms of kinase plasticity. eLife 2014, 3, e04126. [CrossRef]

37. Moore, M.; Shin, M.E.; Bruning, A.; Schindler, K.; Vershon, A.; Winter, E. Arg-Pro-X-Ser/Thr is a consensus phosphoacceptor sequence for the meiosis-specific Ime2 protein kinase in Saccharomyces cerevisiae. Biochemistry 2007, 46, 271-278. [CrossRef]

38. Sawarynski, K.E.; Kaplun, A.; Tzivion, G.; Brush, G.S. Distinct activities of the related protein kinases Cdk1 and Ime2. Biochim. Biophys. Acta 2007, 1773, 450-456. [CrossRef]

39. Sedgwick, C.; Rawluk, M.; Decesare, J.; Raithatha, S.; Wohlschlegel, J.; Semchuk, P.; Ellison, M.; Yates, J., 3rd; Stuart, D. Saccharomyces cerevisiae Ime2 phosphorylates Sic1 at multiple PXS/T sites but is insufficient to trigger Sic1 degradation. Biochem. J. 2006, 399, 151-160. [CrossRef]

40. Shin, M.E.; Skokotas, A.; Winter, E. The Cdk1 and Ime2 protein kinases trigger exit from meiotic prophase in Saccharomyces cerevisiae by inhibiting the Sum1 transcriptional repressor. Mol. Cell. Biol. 2010, 30, 2996-3003. [CrossRef]

41. Sopko, R.; Raithatha, S.; Stuart, D. Phosphorylation and maximal activity of Saccharomyces cerevisiae meiosis-specific transcription factor Ndt80 is dependent on Ime2. Mol. Cell. Biol. 2002, 22, 7024-7040. [CrossRef] [PubMed]

42. Hutchison, E.A.; Glass, N.L. Meiotic regulators Ndt80 and ime2 have different roles in Saccharomyces and Neurospora. Genetics 2010, 185, 1271-1282. [CrossRef] [PubMed]

43. Hutchison, E.A.; Bueche, J.A.; Glass, N.L. Diversification of a protein kinase cascade: IME-2 is involved in nonself recognition and programmed cell death in Neurospora crassa. Genetics 2012, 192, 467-482. [CrossRef] [PubMed]

44. Bielska, E.; Higuchi, Y.; Schuster, M.; Steinberg, N.; Kilaru, S.; Talbot, N.J.; Steinberg, G. Long-distance endosome trafficking drives fungal effector production during plant infection. Nat. Commun. 2014, 5, 5097. [CrossRef] 
45. Garrido, E.; Pérez-Martín, J. The crk1 gene encodes an Ime2-related protein that is required for morphogenesis in the plant pathogen Ustilago maydis. Mol. Microbiol. 2003, 47, 729-743. [CrossRef]

46. Garrido, E.; Voß, U.; Müller, P.; Castillo-Lluva, S.; Kahmann, R.; Pérez-Martín, J. The induction of sexual development and virulence in the smut fungus Ustilago maydis depends on Crk1, a novel MAPK protein. Genes Dev. 2004, 18, 3117-3130. [CrossRef]

47. Higuchi, Y. Initial fungal effector production is mediated by early endosome motility. Commun. Integr. Biol. 2015, 8, e1025187. [CrossRef]

48. Sartorel, E.; Pérez-Martín, J. The distinct interaction between cell cycle regulation and the widely conserved morphogenesis-related (MOR) pathway in the fungus Ustilago maydis determines morphology. J. Cell Sci. 2012, 125, 4597-4608. [CrossRef]

49. Alspaugh, J.A.; Perfect, J.R.; Heitman, J. Cryptococcus neoformans mating and virulence are regulated by the G-protein alpha subunit GPA1 and cAMP. Genes Dev. 1997, 11, 3206-3217. [CrossRef]

50. Kwon-Chung, K.J.; Edman, J.C.; Wickes, B.L. Genetic association of mating types and virulence in Cryptococcus neoformans. Infect. Immun. 1992, 60, 602-605. [CrossRef]

51. Moore, T.D.; Edman, J.C. The alpha-mating type locus of Cryptococcus neoformans contains a peptide pheromone gene. Mol. Cell. Biol. 1993, 13, 1962-1970. [CrossRef] [PubMed]

52. Fraser, J.A.; Subaran, R.L.; Nichols, C.B.; Heitman, J. Recapitulation of the sexual cycle of the primary fungal pathogen Cryptococcus neoformans var. gattii: Implications for an outbreak on Vancouver Island, Canada. Eukaryot. Cell 2003, 2, 1036-1045. [CrossRef]

53. Kim, M.S.; Kim, S.Y.; Jung, K.W.; Bahn, Y.S. Targeted gene disruption in Cryptococcus neoformans using double-joint PCR with split dominant selectable markers. Methods Mol. Biol. 2012, 845, 67-84. [PubMed]

54. Liu, K.H.; Yeh, Y.L.; Shen, W.C. Fast preparation of fungal DNA for PCR screening. J. Microbiol. Methods 2011, 85, 170-172. [CrossRef] [PubMed]

55. Won, M.; Hoe, K.L.; Cho, Y.S.; Song, K.B.; Yoo, H.S. DNA-induced conformational change of Gaf1, a novel GATA factor in Schizosaccharomyces pombe. Biochem. Cell Biol. 1999, 77, 127-132. [CrossRef]

56. Kim, L.; Hoe, K.L.; Yu, Y.M.; Yeon, J.H.; Maeng, P.J. The fission yeast GATA factor, Gaf1, modulates sexual development via direct down-regulation of ste11+ expression in response to nitrogen starvation. PLoS ONE 2012, 7, e42409. [CrossRef]

57. Kmetzsch, L.; Staats, C.C.; Simon, E.; Fonseca, F.L.; Oliveira, D.L.; Joffe, L.S.; Rodrigues, J.; Lourenco, R.F.; Gomes, S.L.; Nimrichter, L.; et al. The GATA-type transcriptional activator Gat1 regulates nitrogen uptake and metabolism in the human pathogen Cryptococcus neoformans. Fungal Genet. Biol. 2011, 48, 192-199. [CrossRef]

58. Lee, I.R.; Chow, E.W.; Morrow, C.A.; Djordjevic, J.T.; Fraser, J.A. Nitrogen metabolite repression of metabolism and virulence in the human fungal pathogen Cryptococcus neoformans. Genetics 2011, 188, 309-323. [CrossRef]

59. Jung, K.W.; Yang, D.H.; Maeng, S.; Lee, K.T.; So, Y.S.; Hong, J.; Choi, J.; Byun, H.J.; Kim, H.; Bang, S.; et al. Systematic functional profiling of transcription factor networks in Cryptococcus neoformans. Nat. Commun. 2015, 6, 6757. [CrossRef]

60. Bayram, O.; Sari, F.; Braus, G.H.; Irniger, S. The protein kinase ImeB is required for light-mediated inhibition of sexual development and for mycotoxin production in Aspergillus nidulans. Mol. Microbiol. 2009, 71, 1278-1295. [CrossRef]

61. Chen, F.; Chen, X.Z.; Su, X.Y.; Qin, L.N.; Huang, Z.B.; Tao, Y.; Dong, Z.Y. An Ime2-like mitogen-activated protein kinase is involved in cellulase expression in the filamentous fungus Trichoderma reesei. Biotechnol. Lett. 2015, 37, 2055-2062. [CrossRef] [PubMed]

62. Katz, M.E.; Cooper, S. Extreme Diversity in the Regulation of Ndt80-Like Transcription Factors in Fungi. G3 Genes Genomes Genet. 2015, 5, 2783-2792. [CrossRef] [PubMed]

63. Staudt, M.W.; Kruzel, E.K.; Shimizu, K.; Hull, C.M. Characterizing the role of the microtubule binding protein Bim1 in Cryptococcus neoformans. Fungal Genet. Biol. 2010, 47, 310-317. [CrossRef] [PubMed]

64. Haase, S.B.; Wittenberg, C. Topology and control of the cell-cycle-regulated transcriptional circuitry. Genetics 2014, 196, 65-90. [CrossRef]

65. Tobe, B.T.; Kitazono, A.A.; Garcia, J.S.; Gerber, R.A.; Bevis, B.J.; Choy, J.S.; Chasman, D.; Kron, S.J. Morphogenesis signaling components influence cell cycle regulation by cyclin dependent kinase. Cell Div. 2009, 4, 12. [CrossRef] 
66. Novak, B.; Csikasz-Nagy, A.; Gyorffy, B.; Chen, K.; Tyson, J.J. Mathematical model of the fission yeast cell cycle with checkpoint controls at the G1/S, G2/M and metaphase/anaphase transitions. Biophys. Chem. 1998, 72, 185-200. [CrossRef]

67. Kellogg, D.R. Wee1-dependent mechanisms required for coordination of cell growth and cell division. J. Cell Sci. 2003, 116, 4883-4890. [CrossRef]

68. Qiu, L.; Wang, J.J.; Ying, S.H.; Feng, M.G. Wee1 and Cdc25 control morphogenesis, virulence and multistress tolerance of Beauveria bassiana by balancing cell cycle-required cyclin-dependent kinase 1 activity. Environ. Microbiol. 2015, 17, 1119-1133. [CrossRef]

69. Sgarlata, C.; Pérez-Martín, J. Inhibitory phosphorylation of a mitotic cyclin-dependent kinase regulates the morphogenesis, cell size and virulence of the smut fungus Ustilago maydis. J. Cell Sci. 2005, 118, 3607-3622. [CrossRef]

70. Bueno, A.; Richardson, H.; Reed, S.I.; Russell, P. A fission yeast B-type cyclin functioning early in the cell cycle. Cell 1991, 66, 149-159. [CrossRef]

71. Sveiczer, A.; Csikasz-Nagy, A.; Gyorffy, B.; Tyson, J.J.; Novak, B. Modeling the fission yeast cell cycle: Quantized cycle times in wee1-cdc25 $\Delta$ mutant cells. Proc. Natl. Acad. Sci. USA 2000, 97, 7865-7870. [CrossRef] [PubMed]

72. Garcia-Muse, T.; Steinberg, G.; Pérez-Martín, J. Characterization of B-type cyclins in the smut fungus Ustilago maydis: Roles in morphogenesis and pathogenicity. J. Cell Sci. 2004, 117, 487-506. [CrossRef] [PubMed]

73. Guttmann-Raviv, N.; Martin, S.; Kassir, Y. Ime2, a meiosis-specific kinase in yeast, is required for destabilization of its transcriptional activator, Ime1. Mol. Cell. Biol. 2002, 22, 2047-2056. [CrossRef] [PubMed]

74. Mitchell, A.P.; Driscoll, S.E.; Smith, H.E. Positive control of sporulation-specific genes by the IME1 and IME2 products in Saccharomyces cerevisiae. Mol. Cell. Biol. 1990, 10, 2104-2110. [CrossRef] [PubMed]

75. Smith, H.E.; Su, S.S.; Neigeborn, L.; Driscoll, S.E.; Mitchell, A.P. Role of IME1 expression in regulation of meiosis in Saccharomyces cerevisiae. Mol. Cell. Biol. 1990, 10, 6103-6113. [CrossRef] [PubMed]

76. Hoe, K.L.; Won, M.S.; Chung, K.S.; Park, S.K.; Kim, D.U.; Jang, Y.J.; Yoo, O.J.; Yoo, H.S. Molecular cloning of gaf1, a Schizosaccharomyces pombe GATA factor, which can function as a transcriptional activator. Gene 1998, 215, 319-328. [CrossRef]

77. Hwang, L.H.; Seth, E.; Gilmore, S.A.; Sil, A. SRE1 regulates iron-dependent and -independent pathways in the fungal pathogen Histoplasma capsulatum. Eukaryot. Cell 2012, 11, 16-25. [CrossRef] 